\title{
Impact of Ocean Spray on the Dynamics of the Marine Atmospheric Boundary Layer
}

\author{
V. N. Kudryavtsev • V. K. Makin
}

Received: 14 September 2010 / Accepted: 11 May 2011 / Published online: 4 June 2011

(C) The Author(s) 2011. This article is published with open access at Springerlink.com

\begin{abstract}
The impact of ocean spray on the dynamics of the marine near-surface air boundary layer (MABL) in conditions of very high (hurricane) wind speeds is investigated. Toward this end, a model of the MABL in the presence of sea-spume droplets is developed. The model is based on the classical theory of the motion of suspended particles in a turbulent flow, where the mass concentration of droplets is not mandatory small. Description of the spume-droplet generation assumes that they, being torn off from breaking waves, are injected in the form of a jet of spray into the airflow at the altitude of breaking wave crests. The droplets affect the boundary-layer dynamics in two ways: via the direct impact of droplets on the airflow momentum forming the so-called spray force, and via the impact of droplets on the turbulent mixing through stratification. The latter is parametrized applying the Monin-Obukhov similarity theory. It is found that the dominant impact of droplets on the MABL dynamics appears through the action of the 'spray force' originated from the interaction of the 'rain of spray' with the wind velocity shear, while the efficiency of the stratification mechanism is weaker. The effect of spray leads to an increase in the wind velocity and suppression of the turbulent wind stress in the MABL. The key issue of the model is a proper description of the spume-droplet generation. It is shown that, after the spume-droplet generation is fitted to the observations, the MABL model is capable of reproducing the fundamental experimental finding - the suppression of the surface drag at very high wind speeds. We found that, at very high wind speeds, a thin part of the surface layer adjacent to the surface turns into regime of limited saturation with the spume droplets, resulting in the levelling off of the friction velocity and decrease of the drag coefficient as $U_{10}^{-2}, U_{10}$ being the wind speed at 10 -m height.
\end{abstract}

\footnotetext{
V. N. Kudryavtsev

Nansen International Environmental and Remote Sensing Centre (NIERSC),

Russian State Hydrometeorological University (RSHU), Saint Petersburg, Russia e-mail: kudr@rshu.ru

V. K. Makin $(\varangle)$

Royal Netherlands Meteorological Institute (KNMI), De Bilt, The Netherlands

e-mail: makin@knmi.nl
} 
Keywords High wind speeds · Marine atmospheric boundary layer ·

Sea-surface drag $\cdot$ Spume droplets · Wave breaking

\section{Introduction}

The energy exchange at the air-sea interface is one of the major physical processes governing hurricane intensity. The air-sea exchanges of heat, moisture and momentum determine how hurricanes gain their strength and intensity from the ocean (Black et al. 2007). On the other hand these exchanges, of momentum in the first place, determine the response of the ocean, resulting in storm surges, waves and currents. While efforts to forecast hurricane tracks have improved greatly, the ability to forecast hurricane intensity has shown little skill (Black et al. 2007; and references therein). Understanding of the air-sea exchange processes is thus crucial in increasing the quality of tropical cyclone modelling. The present study is aimed towards a better understanding of the exchange of momentum in hurricanes.

There is indirect evidence that, at hurricane wind speeds, the drag coefficient does not increase with increasing wind. Modelling studies of tropical cyclones (e.g. Emanuel 1995) showed that they cannot attain their observed intensity if a traditional parametrization of the drag coefficient is used, and it is necessary ad hoc to reduce the drag. Emanuel (1995) showed that the intensity of hurricanes depends on the ratio of enthalpy to the momentum exchange coefficient, and should be in the range 1.2-1.5 to explain the observed winds. The value exceeds twice the value if a traditional parametrization of the drag coefficient is used.

The saturation of the surface stress at increasing wind speeds is implicitly supported by scatterometer measurements. Donnelly et al. (1999) found a saturation of the C-band backscatter power at the wind speed exceeding $25 \mathrm{~m} \mathrm{~s}^{-1}$. The relation of the backscatter to the wind surface stress is not obvious. However, if such a relation does exist, the saturation of the backscatter at high wind speeds presumes a decreasing surface drag coefficient.

The first experimental evidence of the suppression of the surface drag at hurricane wind speeds was reported by Powell et al. (2003). They found that the drag coefficient levels off and starts to decrease with a further increase in the wind speed above about $34 \mathrm{~m} \mathrm{~s}^{-1}$. This finding was confirmed by Jarosz et al. (2007) who found that the drag coefficient peaks at a wind speed near $32 \mathrm{~m} \mathrm{~s}^{-1}$ and then steadily decreases as the wind speed continues to rise. This is contrary to the traditional parametrizations that predict an increase of the drag coefficient with increasing wind speed. Powell et al. (2003) hypothesized that sea spray could significantly influence the transfer of momentum.

Postulating that ocean spray is responsible for the peculiar behaviour of the drag coefficient at hurricane wind speeds we arrive at a classical problem in two-phase fluid dynamics. The theory of the motion of suspended particles (spray droplets in the studied case) in a turbulent flow of incompressible fluid (air in the studied case) was developed by Barenblatt (1953, 1955) and Kolmogorov (1954). The approach was successfully applied to several two-phase geophysical flows, e.g. dust storms, silts on beaches, ocean floor and river beds, and drifting snow (Barenblatt and Golitsyn 1974; Bridge and Dominic 1984; Bintanja 2000). The essence of the theory is that particles embedded in the fluid flow form a stable stratified layer that dampens the turbulent mixing and results in the acceleration of the flow.

It is rather surprising that the application of the approach to the problem of sea spray in hurricanes was overlooked for quite a long time. The finding of Powell et al. (2003) boosted several theoretical studies aiming at the explanation of the observed reduction of the sea drag. Makin (2005) suggested that, at high wind speeds above $30 \mathrm{~m} \mathrm{~s}^{-1}$, a thin (with thickness less than the significant wave height) air boundary layer adjacent to the surface goes into 
a regime of limited saturation (e.g. Barenblatt 1996) by suspended 'light' sea droplets (the dimensionless fall velocity $\omega=a / \kappa u_{*}<1$, where $a$ is the terminal fall velocity, $\kappa$ is von Karman constant, and $u_{*}$ is the friction velocity). This explains the reduction of the drag coefficient as observed by Powell et al. (2003). However, the assumption that the flow turns into that regime by light droplets near the surface is questionable—-such a regime should exist in the main core of the flow.

Barenblatt et al. (2005) considered a more realistic case assuming that sea droplets are rather large with $\omega \gg 1$. They found that the action of the buoyancy forces results in suppression of the turbulence intensity and sharp flow acceleration in a thin layer below the breaking crests where the droplets are produced. Kudryavtsev (2006) (hereinafter K06) followed in general the approach of Barenblatt and Golitsyn (1974) and developed a more elaborate model. He suggested that the effect of sea droplets on the turbulent marine near-surface air boundary layer (MABL) is similar to the effect of temperature stratification. Since both affect turbulence in a similar manner, through the buoyancy force, K06 suggested adopting the well-established universal functions of Monin-Obukhov similarity theory for the stably stratified boundary layer for the description of the MABL in the presence of spray. This approach was also used by Bye and Jenkins (2006). K06 found that droplets cannot affect the MABL dynamics unless one assumes that they are injected into the airflow at the altitude of breaking wave crests. To take this into account, K06 introduced the volume source of droplets in the conservation equation for spray. This term is proportional to the length of wave breaking fronts and models the generation of spume droplets, which being torn off from wave crests are then injected into the airflow at some altitude. As shown, spume droplets may significantly affect the turbulent mixing at strong wind speeds leading to the acceleration of the near-surface wind speed and a reduction of the surface drag similar to that observed by Powell et al. (2003). However, K06 noticed that results of his model depend significantly on the assumed droplet radius. Drawbacks of such an assumption were recently argued by Kudryavtsev and Makin (2009) (hereinafter KM09). Also, when the effect of droplets on the surface drag becomes significant, the contribution of droplets to the mixture density becomes large and comparable to the air density. In this case, the problem should be investigated in the non-Boussinesq approximation, i.e. accounting for the impact of droplets on the momentum balance of the mixture.

In contrast to the above referred studies, Andreas (2004) ignored the effect of sea spray on the atmospheric stratification and focused on their impact on the momentum balance. He split the total stress into turbulent and spray-supported components. Postulating a heuristic relation for the surface spray flux, and assuming its exponential decay with height, he showed that at high wind speeds the spray reduces the airflow velocity and reduces the surface drag. Andreas points out that this result does not corroborate or argue against the results of Powell et al. (2003), as they analyzed different drag coefficients. Andreas's drag coefficient models the turbulent stress in the spray-generation layer (just above the surface), whereas Powell et al. (2003) considered the turbulent stress well above the surface (in the logarithmic boundary layer) and related it to the wind velocity profile and thus analyzed the 'classical' drag coefficient.

We also mention a pioneering study of the aerodynamic roughness of the sea surface at extreme winds under laboratory conditions as reported by Donelan et al. (2004). They observed a saturation of the surface drag at a wind speed exceeding $33 \mathrm{~m} \mathrm{~s}^{-1}$ but for a different reason than in the open ocean. In the laboratory conditions, spray does not play a significant role in the dynamics of the airflow. The separation of the airflow from continually breaking crests is suggested as a mechanism leading to the reduction of the drag coefficient. 
This mechanism is explained in the theoretical studies by Kudryavtsev and Makin (2007) (hereinafter KM07) and Kukulka et al. (2007).

The aim of the present study is to assess the possible impact of droplets on the MABL dynamics through the following mechanisms: (i) the direct effect of droplets on the momentum conservation of air and droplets mixture, and (ii) the suppression of the turbulent mixing due to the effect of droplets on the atmospheric stratification. To this end, we suggest a revised model of the MABL in the presence of spray, generalizing previous models of the studies by the authors: K06, KM07 and KM09. The revision is motivated by the following reasons: first, the assumption that the mass concentration of droplets is negligibly small may lose its validity at high wind speeds. Therefore, the momentum equation for the water-air mixture should be free of such a restriction. Second, the impact of droplets on the MABL dynamics is determined by their scale/radius. Therefore, a spray model is needed to describe consistently the droplet generation, their distribution over the radius and their effect on the turbulence and the momentum exchange.

\section{Mass and Momentum Balance in the Presence of Ocean Spray}

The governing equations are based on the classical theory of the motion of suspended particles in a turbulent flow of an incompressible fluid developed by Barenblatt $(1953,1955)$ and Kolmogorov (1954). The main assumptions of the theory are: (i) the size of particles is small in comparison with the typical scale of turbulence; (ii) the horizontal velocity of particles coincides with that of air, while the vertical velocity differs from that of air by the terminal fall velocity; (iii) the volume and mass concentration of particles in the air are both small. This permits us to assume that droplets form a continuous distribution of mixture in the air, with density

$$
\rho=\rho_{\mathrm{a}}(1-s)+\rho_{\mathrm{w}} s=\rho_{\mathrm{a}}(1+\sigma s),
$$

where $\rho_{\mathrm{a}}$ and $\rho_{\mathrm{w}}$ are the air and the water densities, $s$ is the volume concentration of droplets, and $\sigma=\left(\rho_{\mathrm{w}}-\rho_{\mathrm{a}}\right) / \rho_{\mathrm{a}}$ is the relative excess of the water density over that of air. The classical theory is based on the Boussinesq approximation, i.e. it is assumed that $\sigma s \ll 1$. Therefore, the impact of particles on the turbulent flow dynamics is accounted for only in the turbulent kinetic energy balance equation via the buoyancy force. In this article, we extend the validity range of the theory assuming that $s \ll 1$, but the mass concentration of droplets $\sigma s$ is not mandatory to be small. Thus, terms of order $\sigma s$ are not neglected in the momentum conservation equation (the non-Bousinesq approximation).

\subsection{Mass and Momentum Conservation}

The governing mass and momentum conservation equations for a two-phase fluid free of the condition $\sigma s \ll 1$ are given in the Appendix by Eqs. 56 and 59. Hereinafter, the vertical coordinate $x_{3}$ used in the Appendix is replaced by $z$, and coordinate $x_{1}$ is directed along the wind direction. In the present study, we use a modified form of the mass and momentum conservation equations that take into account the injection of spume droplets into the airflow at altitudes corresponding to the height of breaking wave crests. The equation for conservation of the droplets concentration is written as

$$
\frac{\partial}{\partial z}\left(\overline{s^{\prime} u_{3}^{\prime}}-a \bar{s}\right)=V_{\mathrm{s}}
$$


where $V_{\mathrm{s}}$ is the 'volume source' of spume droplets-the total volume of droplets injected per unit time in unit volume of the air at height $z$. This term simulates the spume-droplet generation by the wind tearing off crests of breaking waves, and was used to investigate the spray-generation problem (Fairall et al. 1990, 2009; K06; KM09). Droplets, being torn off from breaking waves, are injected into the airflow at the altitude of breaking waves crests, and since scales of breaking waves are different, the generation of droplets is spread over height. As argued by Fairall et al. $(1990,2009)$ and K06, the generation of droplets should be included in the mass conservation equation as the volume source rather than the surface flux.

The droplets being torn off from breaking wave crests also inject momentum into the airflow. Thus, a similar term, the volume source of the droplet momentum (rate of injection of momentum of the droplets in unit volume of the air at height $z$ ), must be included in the momentum conservation equation (see Eq. 51 in the Appendix). In contrast to Eq. 2, such a term has never been taken into account in the momentum conservation equation, and it is included in our model construction. Assuming that the droplets are injected with the air velocity, the modified momentum conservation equation is (see Eq. 59 in the Appendix)

$$
\frac{\partial}{\partial z}\left(\bar{\rho} \overline{u_{1}^{\prime} u_{3}^{\prime}}\right)=\rho_{\mathrm{w}} F_{\mathrm{s}} \frac{\partial U}{\partial z}
$$

where $U$ is the mean wind velocity, and $F_{\mathrm{s}}$ is the droplet flux $F_{\mathrm{s}}$ defined via $V_{\mathrm{s}}$ as

$$
F_{\mathrm{s}}=\int_{z}^{\infty} V_{\mathrm{s}} \mathrm{d} z .
$$

The term on the right-hand side (r.h.s.) of (3) describes the 'vortex' force acting on the airflow due to the vertical flux of droplet ('rain' of spray) through the velocity shear. Anticipating that the droplet flux is compensated by their falling (i.e. $F_{\mathrm{S}} \approx a \bar{s}$, see Sect. 2.2.1 below), this term implies that droplets moving downwards transfer the momentum from the upper layers of higher velocity to the lower layers of lower velocity, which results in acceleration of the airflow in the spray-generation layer. In Eqs. 2 and $3, \overline{s^{\prime} u_{3}^{\prime}}$ and $\overline{u_{1}^{\prime} u_{3}^{\prime}}$ are the vertical turbulent kinematic fluxes of droplets and momentum. Hereinafter, the overbar in the mean density $\bar{\rho}$ and the mean concentration $\bar{s}$ is omitted.

Based on the argumentation by K06, we consider Eqs. 2 and 3 as mass and momentum conservation equations averaged in the coordinate system that follows the sea surface formed by the dominant surface waves. Short waves in the tail of the wave spectrum support the major part of the sea drag due to the wave-induced momentum flux and the airflow separation (see, e.g. KM07). Therefore, in the momentum balance equation (3), they are parameterized through the aerodynamic roughness length $z_{0}$. On the other hand, KM09 showed that most droplets are produced by the wind tearing off crests of the shortest breaking waves. Therefore, the effect of droplets torn off from short breaking waves is included in the mass and momentum conservation equations (2) and (3) through the volume source $V_{\mathrm{s}}$.

The solution of Eq. 2 depends on the size of droplets through the terminal fall velocity $a$. Therefore, we consider the mass conservation equation (2) for the spectral concentration, i.e. for the concentration of droplets with radius in the range $r$ to $r+\mathrm{d} r$. Hereinafter, the hat over any quantity $Y$ denotes its spectral density (the distribution over the droplet radii). If the spectral density $\hat{Y}$ of a quantity $Y$ is defined, then its total value is $Y=\int \hat{Y} \mathrm{~d} r$. Assuming that far enough from the sea surface $\hat{s}$ is negligible, Eq. 2 in the spectral form reads

$$
-\hat{q}_{\mathrm{s}}+a \hat{s}=\hat{F}_{\mathrm{s}}
$$


where $\hat{s}$ is the droplet volume concentration spectrum-the volume of droplets of radius $r$ per unit volume of air (units $\mathrm{m}^{3} \mathrm{~m}^{-3} \mu \mathrm{m}^{-1}$ ), $q_{\mathrm{s}} \equiv \overline{s^{\prime} u_{3}^{\prime}}$ is the total turbulent flux of droplets and $\hat{q}_{\mathrm{s}}$ is the spectral flux of droplets of radius $r$, and $\hat{F}_{\mathrm{s}}$ (units $\mathrm{m}^{3} \mathrm{~m}^{-2} \mathrm{~s}^{-1} \mu \mathrm{m}^{-1}$ ) is the spectrum of the total volume droplet flux $F_{\mathrm{S}}$ defined by (4). It is noted that the quantity $\hat{F}_{\mathrm{S}}$ is often called the spray-generation function.

In contrast to the classical theory of the turbulent surface boundary layer, Eq. 3 does not predict the constancy of the turbulent momentum flux over height. Well above the sea surface, the effect of droplets on airflow dynamics is negligible, so that $\rho=\rho_{\mathrm{a}}$, and the turbulent momentum flux is $\rho \overline{u_{1}^{\prime} u_{3}^{\prime}}=-\rho_{\mathrm{a}} u_{*}^{2}$, where hereinafter $u_{*}$ is the friction velocity outside the layer influenced by droplets. Equation 3 can be written as

$$
\rho_{\mathrm{a}} u_{*}^{2}=\rho v_{*}^{2}-\rho_{\mathrm{w}} \int_{z}^{\infty} F_{\mathrm{s}}(\partial U / \partial z) \mathrm{d} z
$$

where $\rho v_{*}^{2}=-\rho \overline{u_{1}^{\prime} u_{3}^{\prime}}$ is the local turbulent stress. Since the second term on the r.h.s. is positive, this equation predicts an increase of the turbulent stress in the spray-generation layer. Equation 6 is evidently equivalent to

$$
\rho_{\mathrm{a}} u_{*}^{2}=\rho v_{*}^{2}+\rho_{\mathrm{w}} F_{\mathrm{s}} U-\rho_{\mathrm{w}} \int_{z}^{\infty} V_{\mathrm{s}} U \mathrm{~d} z .
$$

If we omit the last term on the r.h.s., then we arrive at the momentum conservation equation suggested by Andreas (2004, his Eq. 3.1), where the second term is the 'spray stress'. Andreas (2004) assumed that the droplets appear in the airflow in a 'state of rest', and therefore this spray stress corresponds to the momentum that the droplets extract from the airflow to be accelerated to the wind velocity. In this case, the total momentum flux is distributed between the air and the droplets, and thus, the turbulent stress in the spray-generation layer is reduced. However, as shown in Andreas (2004), the effect of spray results in an increase in the turbulent stress above the spray-generation layer as compared with the background (no spray effect) value.

According to the model of this study, the droplets are accelerated to the wind speed instantaneously at generation. Once generated, the droplets are injected into the airflow with velocity equal to the wind velocity at the altitude of breaking crests, i.e. they do not need to be accelerated. The rate of the integral input of the momentum due to injection of the droplets into the airflow is described by the last term in (7). The balance between the rate of the momentum input into the layer above a given level $z=$ constant and the sink of the momentum due to downward spray momentum flux through the surface $z=$ constant (second term on r.h.s. of (7)) results in the accumulation of momentum in this layer, i.e. acceleration of the airflow. However, under the assumption of stationarity, the net momentum gain must be compensated by enhanced turbulent shear stress described by the first term on the r.h.s. of (7). At the sea surface, this shear stress is

$$
\left(\rho v_{*}^{2}\right)_{z=0}=\rho_{\mathrm{a}} u_{*}^{2}+\rho_{\mathrm{w}} \int_{0}^{\infty} V_{\mathrm{s}} U \mathrm{~d} z
$$

where we took into account that $U(0)=0$. The first term on the r.h.s. of (8) is associated with the form drag of the sea surface, and the second with the total force required to tear off the droplets from breaking crests and to accelerate them to the wind velocity, and then to 
inject them into the airflow. Notice that the enhanced stress $\rho v_{*}^{2}(0)$ acts on the lower part of the atmospheric boundary layer, while the stress acting on the water side is equal to $\rho_{a} u_{*}^{2}$.

KM07 showed that the aerodynamic roughness length $z_{0}$ of the sea surface under high wind speed conditions can be parametrized through

$$
z_{0}=c_{*} u_{*}^{2} / g
$$

where $c_{*}$ is the Charnock parameter. At wind speeds up to $u_{10}=20 \mathrm{~m} \mathrm{~s}^{-1} c_{*}$ grows due to the strong wind-speed dependence of the airflow separation, but at higher wind speeds $c_{*}$ is saturated due to the sheltering effect. Focusing on high wind conditions we follow KM07 and fix $c_{*}$ at a constant value $c_{*}=0.014$. It is noted that, following the above reasoning, we defined $z_{0}$ via the friction velocity $u_{*}$ outside the layer directly affected by droplets.

\subsubsection{Closure Hypotheses}

Based on the classical closure for the turbulent flow with suspended particles, mass and momentum fluxes in (2) and (3) are defined, respectively, as

$$
\begin{gathered}
\tau \equiv-\rho \overline{u_{1}^{\prime} u_{3}^{\prime}}=\rho K \frac{\partial U}{\partial z}, \\
-q_{\mathrm{s}} \equiv-\overline{s^{\prime} u_{3}^{\prime}}=K \frac{\partial s}{\partial z},
\end{gathered}
$$

where $K$ is the turbulent eddy viscosity, which is assumed to be the same for the turbulent diffusion of droplets. As argued by K06, the effect of sea droplets and the thermal stratification on turbulence appears additively. Therefore, results of the Monin-Obukhov similarity theory for the non-neutral boundary layer can be applied to the boundary layer stratified due to presence of sea droplets. In this case, the turbulent eddy viscosity reads

$$
\begin{aligned}
& K=\kappa\left(z+z_{0}\right) v_{*} / \Phi, \\
& \Phi\left(z / L_{\mathrm{s}}\right)=1+b z / L_{\mathrm{s}},
\end{aligned}
$$

where $1 / L_{\mathrm{s}}=\kappa g\left(\Delta \rho / \rho_{\mathrm{a}}\right) q_{\mathrm{s}} / v_{*}^{3}$ is the inverse Obukhov length for the turbulent flow stratified due to the presence of droplets, and $b=5$ is an empirical constant. Notice that both the eddy viscosity and the Obukhov length are dependent on the local friction velocity $v_{*}=(\tau / \rho)^{1 / 2}$. Using (5), the inverse Obukhov length is defined as

$$
\frac{1}{L_{\mathrm{s}}}=\kappa \frac{\Delta \rho}{\rho_{\mathrm{a}}} \frac{g}{v_{*}^{3}} \int\left(a \hat{s}-\hat{F}_{\mathrm{s}}\right) \mathrm{d} r .
$$

The turbulent eddy viscosity (12) with the use of closure (10) can be expressed through the mean wind velocity shear as

$$
K=\frac{\kappa^{2}\left(z+z_{0}\right)^{2}}{\Phi^{2}} \frac{\partial U}{\partial z} .
$$

\subsection{Wind and Droplet Profiles and the Sea Drag}

Let us now find the solution of the problem described by the governing equations (3) and (5). First we mention that at high wind speeds the density of the air-droplet mixture $\rho$ on the left-hand side of Eq. 3 may be different from the air density $\rho_{\text {a }}$. However, the impact of this deviation, $\Delta \rho s v_{*}^{2}$, on the solution is insignificant as compared with the impact of the vortex force described by the r.h.s. of (3). It can be shown that the ratio of these terms is of order 
$v_{*}^{2} /(a U)$, which is very small because in the range of spume droplets $v_{*} / a$ is of order $10^{-1}$ (see e.g. Fig. 2 from K06).

Thus, ignoring the deviation of $\rho$ from $\rho_{\mathrm{a}}$ and using the closure scheme (10)-(15), the governing equations (3) and (5) take the form

$$
\frac{\partial^{2} U}{\partial z^{2}}+\frac{\Phi}{\left(z+z_{0}\right)} \frac{\partial}{\partial z}\left(\frac{z+z_{0}}{\Phi}\right) \frac{\partial U}{\partial z}+\frac{\sigma F_{\mathrm{S}} \Phi^{2}}{2 \kappa^{2}\left(z+z_{0}\right)^{2}}=0
$$

for momentum, and

$$
\frac{\partial \hat{s}}{\partial z}=-\frac{\omega \Phi}{z+z_{0}}\left(\hat{s}-\hat{s}_{*}\right)
$$

for the droplet concentration. Hereinafter, $\omega=a /\left(\kappa v_{*}\right)$ is the dimensionless terminal fall velocity scaled by the local friction velocity, and $\hat{s}_{*}=\hat{F}_{\mathrm{S}} / a$ is the scale of the droplet concentration. The boundary conditions for (16) are $U=0$ at $z=0$ and $U=U_{h}$ at $z=h$ where $U_{h}$ is wind speed at the reference level $h$. The boundary condition for (17) is specified on the sea surface $z=0$ as $\partial s / \partial z=0$, which is equivalent to specifying $\hat{s}(0)=\hat{s}_{*}(0)$.

The solution of Eq. 16 obeying the boundary conditions can be written as

$$
U(z)=\frac{u_{*}}{\kappa} \int_{0}^{z} \Phi \mathrm{d} \zeta+\int_{0}^{z}\left(u_{\mathrm{s}}^{h}-u_{\mathrm{s}}\right) \Phi \mathrm{d} \zeta
$$

where $\zeta=\ln \left(z+z_{0}\right)$ is the integration variable, $u_{\mathrm{s}}$ is the 'spray-forced wind velocity' defined by

$$
u_{\mathrm{s}}(z)=\left(2 \kappa^{2}\right)^{-1} \sigma \int_{0}^{z} F_{\mathrm{s}} \Phi \mathrm{d} \zeta
$$

$u_{\mathrm{s}}^{h}=u_{\mathrm{s}}(h)$, and $u_{*}$ is the friction velocity outside the spray-generation layer defined by the following resistance law:

$$
C_{\mathrm{d} h} \equiv\left(u_{*} / U_{h}\right)^{2}=\kappa^{2}\left[\int_{0}^{h} \Phi \mathrm{d} \zeta+\left(\kappa / u_{*}\right) \int_{0}^{h}\left(u_{\mathrm{s}}^{h}-u_{\mathrm{s}}\right) \Phi \mathrm{d} \zeta\right]^{-2},
$$

where $C_{\mathrm{d} h}$ is the drag coefficient for the reference level $z=h$.

Well above the droplet-generation layer (where $F_{\mathrm{s}}$ vanishes) the effect of the spray-forced velocity on the wind profile (described by the second term on the r.h.s. of (18)) disappears. Moreover spume droplets are 'heavy' and tend to fall back to the surface once generated. Therefore, it could be anticipated that well above the spray-generation layer the concentration of droplets is so small that $z / L_{\mathrm{S}} \ll 1$ and therefore $\Phi \simeq 1$. Thus, in this case droplets do not locally affect the wind-velocity profile, and (18) turns into the standard logarithmic form. Inside the spray-generation layer (where the balance $a \hat{s} \approx \hat{F}$ is approximately fulfilled, and thus, $z / L_{\mathrm{s}} \ll 1$, see (14)) the stratification function $\Phi$ is again $\Phi \approx 1$. However, the efficiency of the spray-forced mechanism (described by the second term in (18)) in this layer is maximal and the impact of this factor results in the increase of the wind velocity in the spray-generation layer. As mentioned above, the physical reason for such an acceleration is the action of the vortex force resulting from the interaction of the 'rain of spray' with the velocity shear. 
The effect of spray leads to the suppression of the turbulent mixing above the spraygeneration layer as predicted by the resistance law (20). This equation clearly shows that both the effect of spray on the atmospheric stratification (the stratification function is $\Phi>1$ ) and the spray-force effect results in a decrease in the drag coefficient. However, the efficiency of this effect depends on the magnitude of the spray-generation function and on the spectral distribution of the droplets over the size; this will be analyzed below.

Within the framework of our closure scheme, the local friction velocity is defined as: $v_{*}=\kappa\left(z+z_{0}\right) \Phi^{-1} \partial U / \partial z$, and with the use of (18) its vertical distribution is

$$
v_{*}(z)=u_{*}+\kappa\left[u_{\mathrm{s}}^{h}-u_{\mathrm{s}}(z)\right] .
$$

Outside the spray-generation layer $v_{*}=u_{*}$, and at the surface the local friction velocity exceeds $u_{*}$. The difference $v_{*}^{2}(0)-u_{*}^{2}$ corresponds to the force required to accelerate the droplets (torn off from all breaking crests) to the airflow velocity and to inject them into the air. As noted above, the enhanced stress $\rho v_{*}^{2}(0)$ acts only on the lower part of the air boundary layer, while the stress acting on the water side (below the surface) is equal to $\rho_{\mathrm{a}} u_{*}^{2}$.

Equations 18 and 20 define the problem if the distribution of the droplet concentration $\hat{s}(z, r)$ with height and over size is known. For that, we need to solve Eq. (17) obeying the surface boundary condition $\partial \hat{s} / \partial z=0$ at $z=0$. The solution reads

$$
\hat{s}(z, r)=\hat{s_{*}}(z, r)+\int_{0}^{z} \exp \left[-\int_{z^{\prime}}^{z} \omega \Phi \mathrm{d} \zeta\right] a^{-1} \hat{V}_{\mathrm{s}} \mathrm{d} z^{\prime} .
$$

\subsubsection{Asymptotic Solution}

The spume droplets are 'heavy' and characterized by large values of the dimensionless fall velocity, $\omega \gg 1$ (see, e.g. Fig. 2 from K06). In this case, Eq. 22 has a remarkable solution, which approximately reads

$$
\hat{s}(z) \approx \hat{F}_{\mathrm{S}}(z) / a
$$

and states that the droplet concentration results from the balance of the droplet flux and the gravitational force; the vertical transport by turbulence being inefficient. The spray-generation function $F_{\mathrm{S}}\left(u_{*}, z\right)$ can be represented as $F_{\mathrm{S}}\left(u_{*}, z\right)=F_{\mathrm{s}}^{0}\left(u_{*}\right) f_{\mathrm{s}}(z / \delta)$ where $F_{\mathrm{S}}^{0}\left(u_{*}\right)$ is the flux of spume droplets at the surface (depending on the wind speed only) and $f_{\mathrm{s}}(z / \delta)$ describes the vertical profile of the spume-droplet generation ( $\delta$ is the depth scale of the droplet-generation layer). Taking into account the balance (23) one may anticipate that the stratification function $\Phi$ (13) with (14) is about $\Phi \approx 1$. Then the wind profile (18) with (19) approximately reads

$$
\kappa U(z) / u_{*}=\ln \left(\frac{z+z_{0}}{z_{0}}\right)+\frac{\sigma F_{\mathrm{s}}^{0}}{2 \kappa u_{*}} \int_{0}^{z}\left(\int_{z}^{h} f_{\mathrm{s}}\left(z^{\prime} / \delta\right) \mathrm{d} \zeta^{\prime}\right) \mathrm{d} \zeta,
$$

where we repeat that $\zeta=\ln \left(z+z_{0}\right)$ is an integration variable. Both the model and empirical relations predict a strong dependence of $F_{\mathrm{s}}^{0}$ on the wind speed; in particular $F_{\mathrm{s}}^{0} \sim u_{*}^{4}$ follows from the empirical spray-generation function for spume droplets by Andreas (1998) (shown in Fig. 2 by K06), and from the model of KM09, their Eq. 21. In this case, the wind velocity in the whole layer increases and its deviation from the reference no-spray wind profile is strongly wind dependent, as $u_{*}^{3}$. 
For this case, the surface drag defined by (20) can be rewritten as

$$
C_{\mathrm{d} h}=\kappa^{2}\left[\ln \left(\frac{h}{z_{0}}\right)+\frac{\sigma F_{\mathrm{s}}^{0}}{4 \kappa^{2} u_{*}} \ln ^{2}\left(\frac{\delta}{z_{0}}\right)\right]^{-2},
$$

where we assume that the reference level $h$ is above the spray-generation layer, i.e. $h>\delta$, and to derive (25) we took into account that the integral $\int_{z}^{h} f_{\mathrm{s}}(z / \delta) \mathrm{d} \zeta$ in (24) can be evaluated as: 0 if $z>\delta$, and $\ln (\delta / z)$ if $z<\delta$. If, as before, we suggest that $F_{\mathrm{s}}^{0} \sim u_{*}^{4}$ then Eq. 25 predicts a decreasing drag coefficient with increasing wind speed. The term describing the impact of spume droplets on $C_{\mathrm{d} h}$ increases very rapidly with the increasing wind speed, as $u_{*}^{3}$. However, the significance of this impact thoroughly depends on the magnitude of the spray-generation function $F_{\mathrm{s}}^{0}$ at the surface.

\section{Effect of Spray Specified Empirically}

To close the problem and assess the impact of spray on the boundary-layer dynamics one needs to define the spray-generation function $F_{\mathrm{S}}(z)$ (or the scale $\hat{s}_{*}=\hat{F}_{\mathrm{S}} / a$ ), or the volume source $V_{\mathrm{S}}$ linked to $F_{\mathrm{S}}$ by (4). In this section, for illustrative purpose, we consider a qualitative behaviour of the coupled system (18), (20) and (22), when $F_{\mathrm{S}}(z)$ is specified empirically according to Andreas (1998, 2004). Following Andreas (2004) the volume flux of droplets $F_{\mathrm{s}}$ is specified as

$$
F_{\mathrm{S}}(z)=c_{s} u_{*}^{4} \exp (-z / \delta)
$$

where $c_{s}$ is a dimensional constant, $\delta=H_{\mathrm{S}} / 7, H_{\mathrm{S}}$ is the significant wave height, and $u_{*}$ is the friction velocity well above $H_{\mathrm{s}}$. In the present calculations, the significant wave height is specified as: $H_{\mathrm{s}}=2.910^{-3} \widetilde{x}^{0.45} u_{10}^{2} / g$, where $\tilde{x}=X g / U_{10}^{2}$ is the dimensionless fetch (Komen et al. 1994). It is noted that the wind-speed dependence of $F_{\mathrm{s}}$ defined by (26) results from the numerical calculations of the spectral spray-generation function originally suggested by Andreas (1998) in the range of droplet radii $r>20 \mu \mathrm{m}$, corresponding to the spume droplets (see also Fig. $2 \mathrm{~b}$ from K06). In order to fit $F_{\mathrm{s}}$ at the surface to the empirical function, the constant $c_{s}$ in (26) must equal $5 \times 10^{-7}$ with the dimension $\left[\mathrm{m}^{-3} \mathrm{~s}^{3}\right]$. The vertical profile of $F_{\mathrm{s}}$ in (26) was originally proposed by Andreas (2004) for the droplet concentration. However, anticipating that in the spray-generation layer the balance of spume droplets approximately reads $F_{\mathrm{S}} \approx a s$, we assume that the vertical distribution of the spray concentration and the rate of its production should have a similar shape.

The spectral shape of the empirical spray-generation function in the range of spumedroplet radii from $r=20$ to $r=100 \mu \mathrm{m}$ is proportional to $\propto r^{2}$ (see, e.g. Figs. 2a, b from KM09). Therefore, for the sake of simplicity we suggest that the spectral distribution of $F_{\mathrm{S}}$ defined by (26) has the shape $3 r^{2} / r_{0}^{3}$, where $r_{0}$ is the spectral cut-off of the spray-generation function. To simulate the empirical spray-generation function proposed by Andreas (2004) the spectral cut-off should be chosen at $r_{0}=200 \mu \mathrm{m}$.

The calculations discussed below are performed for a fetch $X=500 \mathrm{~km}$. It was found that the effect of the droplets defined by the spray-generation function (26) with the spectral cut-off at $r_{0}=200 \mu \mathrm{m}$ on the MABL is negligible and thus, not shown here. Andreas (2004) came to a similar conclusion considering the impact of spray on the MABL via 'the spray-stress' effect. To magnify the effect, he suggested a heuristic spray- 
generation function, which has the same form as (26) but with the constant $c_{s}$ amplified 10 times.

In this study, the spectral spray-generation function is used. It could be anticipated that the main drawback of the empirical spray-generation function is a lack of its knowledge for the largest generated droplets. The cut-off of the empirical function at $r_{0}=200 \mu \mathrm{m}$ presumes that the data used for the construction of this function did not contain reliable information on the statistics of the droplets of larger radius, because they are heavy (and thus, not transported by turbulence upward but fall down to the surface once generated) and were not traced at the altitude of measurements. Therefore, we suggest increasing the cut-off radius, keeping however the spray-generation-function spectrum in the range $10<r<200 \mu \mathrm{m}$ on the original empirical level. Extrapolation of the spray-generation-function spectrum towards larger radii presumes that the proportionality constant $c_{s}$ in the integral $F_{\mathrm{S}}$ defined by (26) depends on the choice of the new cut-off radius $r_{0 n}$ and reads $c_{s}=5 \times 10^{-7} r_{0 n}^{3} / r_{0}^{3}$, where $r_{0}=200 \mu \mathrm{m}$ is the original cut-off.

Following the experimental results of Koga (1981), Anguelova et al. (1999) and Fairall et al. (2009) we assume that the maximum radius of spume droplets is $\mathrm{O}(1000) \mu \mathrm{m}$. The model calculations with $r_{0}$ taken at $750 \mu \mathrm{m}$ are shown in Fig. 1 for the wind speed $U_{h}=70 \mathrm{~m} \mathrm{~s}^{-1}$ specified at the reference level $h=100 \mathrm{~m}$. Notice that, in specifying $c_{s}$ and $r_{0}$, we do not intend to fit the model results to any observations because these calculations are performed for the illustrative purpose only.

The solid line in Fig. 1a shows the model wind profile when the effect of droplets on the MABL dynamics is taken into account, and the dashed line shows the background wind profile (no spray effect). The presence of droplets results in the acceleration of the airflow. The dimensionless wind-velocity shear defined from (18) as

$$
\frac{\kappa z}{u_{*}} \frac{\partial U}{\partial z}=\frac{\Phi}{1+z_{0} / z}\left[1+\frac{\kappa}{u_{*}}\left(u_{\mathrm{s}}^{h}-u_{\mathrm{s}}\right)\right]
$$

is shown in Fig. 1b. The first term on the r.h.s. of (27) describes the effect of the droplets on the wind-velocity shear via the stratification, and the second one via the spray-force effect. The solid line in Fig. 1b shows the model profile when both the effect of the spray force and the effect of droplets on the stratification are included. The effect of droplets via the stratification results in an increase of the wind-velocity shear in the layer above the spraygeneration layer only, while their effect via the spray-force effect results in the increase of the velocity shear in the whole spray-generation layer. Referring to Fig. 1b, one may conclude that the effect of the spray force dominates the airflow acceleration. Figure 1c shows the profile of the turbulent stress scaled by its reference (no spray effect) value. Above the spray-generation layer the turbulent stress is decreased by a factor of two while at the sea surface (more precisely, at the lower boundary of the MABL) it is enhanced by a factor of two relative to the reference value. The latter results from the action of the force required to tear off the droplets from breaking crests and to accelerate them to the wind velocity. Being injected at the altitude of breaking crests, the droplets then are falling and accelerate the airflow in the spray-generation layer due to the action of the spray-vortex force. This acceleration in turn results in a decrease in the wind velocity shear above the spraygeneration layer, and thus, to suppression of the turbulence and the turbulent stress in this layer.

Figure 1d shows the drag coefficient at 10-m height defined as $C_{d 10}=\left(u_{*} / U_{10}\right)^{2}$. When the effect of droplets on the drag coefficient via stratification (described by the first term in (20)) is only taken into account, $C_{d 10}$ decreases as compared to the reference run, though the magnitude of the effect is not too significant. When the 

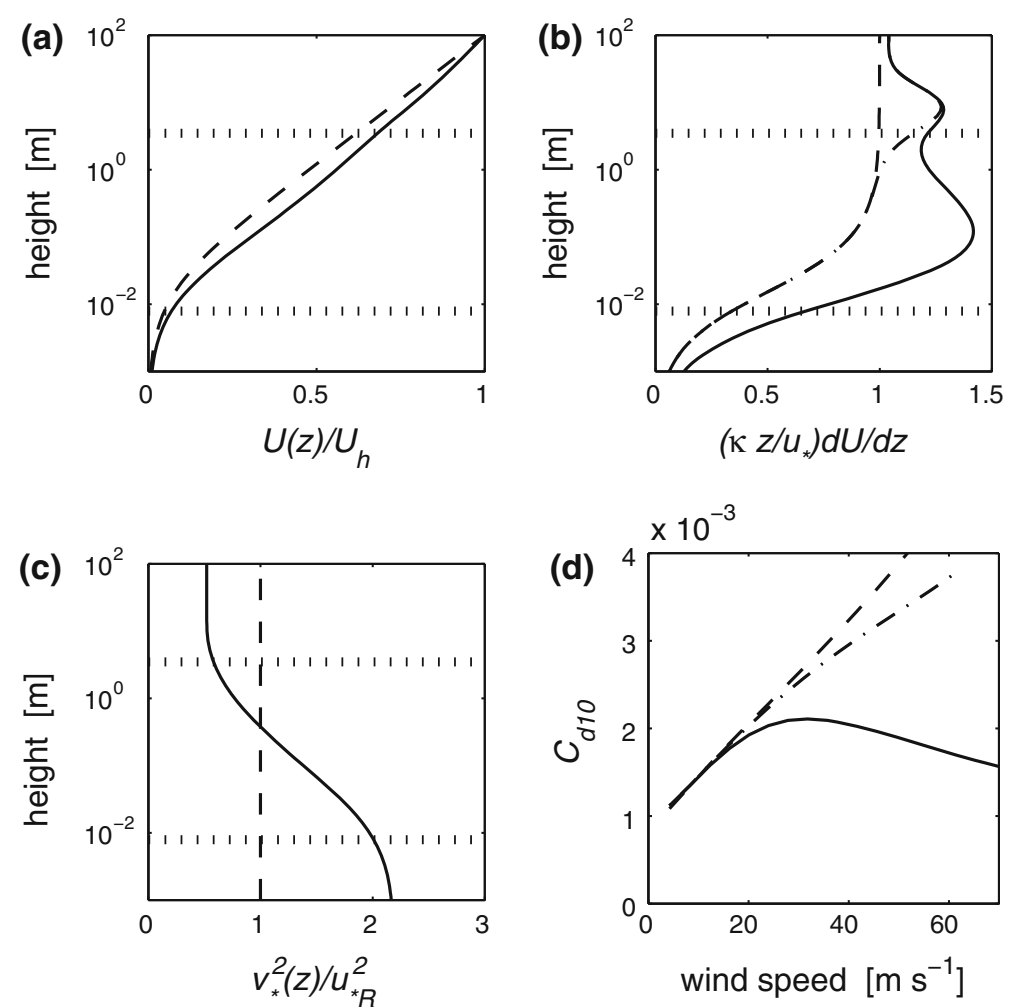

Fig. 1 a The wind profile $U(z) / U_{h}$, where the wind speed at the reference level $h=100 \mathrm{~m}$ is $U_{h}=70 \mathrm{~m} \mathrm{~s}^{-1}$ $\left(U_{10}=55 \mathrm{~m} \mathrm{~s}^{-1}\right)$, solid line effect of spray is accounted for, dashed line the reference run (no spray effect); b The dimensionless wind-shear profile defined by (27), line types: dashed line the reference run, dash-dotted effect of stratification is taken only into account (first term on the r.h.s. of (27)), solid line both the stratification and the spray-force effects are taken into account; $\mathbf{c}$ The kinematic stress $v_{*}^{2} / u_{* R}^{2}$ profile defined by (21) and scaled by the reference (without spray) friction velocity $u_{* R}$; d The drag coefficient at 10 -m height versus the wind speed $U_{10}$; line types as in plot (b). Horizontal dotted lines in plots a-c indicate surface roughness length $z_{0}$ (thin dotted) and depth $\delta$ of the spray-generation layer (thick dotted)

effect of droplets on $C_{d 10}$ through the 'spray force' (described by the second term in (20)) is accounted for, a strong reduction of $C_{d 10}$ relative to the reference values is found. This result apparently follows from the approximate solution (25) that predicts fast enhancement of the impact of spray on surface drag with increasing wind speed $\left(\sim u_{*}^{3}\right)$.

Thus, droplets can affect the MABL dynamics in two ways: by changing the stratification of the MABL, and by the effect of spray on the mixture momentum via the effect of the vortex force (the spray-force effect), the latter being much more efficient. The effect of the spray force leads to the acceleration of the airflow and the suppression of the turbulent stresses above the spray-generation layer. The latter is associated with the reduction of the drag coefficient as was observed by Powell et al. (2003). We note however that this conclusion is based on the model calculations with the empirical spray-generation function defined by (26), with the distribution of droplets over the size proportional to $r^{2}$ and where the spectral cut-off was fixed at $r_{0}=750 \mu \mathrm{m}$. 


\section{Effect of Spray: Model Results}

In this section, we present results of the model calculations with a theoretical $F_{\mathrm{S}}(z)$, as suggested by KM09.

\subsection{Model Spray-Generation Function}

According to KM09, the production rate of spume droplets is given by

$$
\left.V_{\mathrm{s}}(z) \propto F_{0 \mathrm{~s}} z^{-2} \Lambda(k)\right|_{k=1 / z},
$$

where $F_{0 \mathrm{~s}}\left(\mathrm{~m}^{3} \mathrm{~m}^{-2} \mathrm{~s}^{-1}\right)$ is the total volume flux of droplets (integrated over the droplet radius) from an individual breaking crest, and $\Lambda(k)$ is length of the breaking crests of waves in the wavenumber range $k$ to $k+\mathrm{d} k$ (integrated over all directions).

Equation 28 describes the production of droplets by the wind tearing off breaking crests. Water/foam on the crest of a breaking wave with wavenumber $k$ is pulverized into droplets, which are then injected into the airflow at the altitude of the breaking crest $z \simeq 1 / k$. The injection of droplets presumes that they are torn off from a breaking crest and are further accelerated to match the airflow velocity $U_{\mathrm{s}}$ in the vicinity of the wave crest. As shown by KM09, the force required to accelerate these droplets to $U_{\mathrm{s}}$ is $\rho_{\mathrm{w}} F_{0 \mathrm{~s}} U_{\mathrm{s}}$ and equals the local turbulent wind stress over the breaking crest that is proportional to $\rho_{\mathrm{a}} U_{\mathrm{s}}^{2}$. Thus, $\rho_{\mathrm{w}} F_{0 \mathrm{~s}} U_{\mathrm{s}} \propto \rho_{\mathrm{a}} U_{\mathrm{s}}^{2}$ and the droplet flux reads

$$
F_{0 \mathrm{~s}} \propto\left(\rho_{\mathrm{a}} / \rho_{\mathrm{w}}\right) U_{\mathrm{s}} .
$$

Equation 29 describes the total production of droplets from an individual breaking crest.

The distribution of droplets over size follows from the balance of the restoring force associated with the surface tension on the droplet surface, and the dynamic pressure force associated with the turbulent velocity differential over the droplet (Kolmogorov 1949). Applying the Kolmogorov-Obukhov theory of the local structure of turbulence for the boundary layer over a breaking crest, where spume droplets are produced, KM09 found that droplets injected into the airflow have the following distribution over size

$$
F_{0 \mathrm{~s}} \propto 3\left(\rho_{\mathrm{a}} / \rho_{\mathrm{w}}\right) U_{\mathrm{s}} r_{0}^{-3} \int_{r<r_{0}} r^{2} \mathrm{~d} r,
$$

where $r_{0}$ is the maximal radius of generated droplets

$$
r_{0} \propto(\gamma v / k)^{1 / 3} u_{*}^{-1},
$$

and where $\gamma$ is the surface tension, and $v$ is the air molecular viscosity coefficient. It is noted that, in order to derive (30), KM09 suggested that the size of the spume droplets corresponds to the dissipation interval of the turbulence. The upper limit of the length scale of the eddies belonging to this interval is of order $10 \eta$ where $\eta=\left(v^{3} / u_{*}^{3} / k\right)^{1 / 4}$ is the Kolmogorov length scale. For wavenumbers of the shortest breaking waves between 5 and $25 \mathrm{radm}^{-1}$ (see Sect. 4.1 .1 below) and $u_{*}=1 \mathrm{~ms}^{-1}$, the upper limit of the dissipation interval is between $10^{-3}$ and $1.5 \times 10^{-3} \mathrm{~m}$, which is consistent with the maximum size of the considered spume droplets.

A specific distribution of length of the wave breaking crests is an open question. However, field studies showed that $\Lambda(\mathbf{k})$ can be parametrized as (Melville and Matusov 2002; Gemmrich et al. 2008)

$$
\Lambda(\mathbf{k}) \propto k^{-1}\left(u_{*} / c\right)^{3},
$$


which is valid in the range of $k$ from the spectral peak wavenumber $k_{\mathrm{p}}$ to the wavenumber of the shortest breaking waves $k_{\mathrm{b}}$ producing measured white caps. As follows from (28) and (32), the production rate of droplets is supported mainly by short breaking waves, and attenuates rapidly with height.

The droplet flux $F_{0 \mathrm{~s}}$ is defined by (30), and depends on $z$ via the velocity scale $U_{s}$ and the maximum radius of generated droplets $r_{0}$, defined by (31). However, since most droplets are produced by the breaking of short waves at low altitudes, we may ignore the dependence of $F_{0 \mathrm{~s}}$ and $r_{0}$ on $z$, defining them at $z=1 / k_{\mathrm{b}}$, where $k_{\mathrm{b}}$ is the wavenumber of the shortest breaking waves producing spume droplets. Furthermore, we suggest that the velocity scale $U_{\mathrm{s}}$ acting on the crest of a breaking wave is proportional to the mean wind speed at $z=1 / k_{\mathrm{b}}$, which can be scaled by the air friction velocity at the surface $v_{*}^{0}=v_{*}(0)$. It is noted that when the effect of droplets on MABL becomes strong, the acceleration of the airflow leads to the enhanced production of spume droplets, and this effect is properly reproduced by the scaling of $U_{\mathrm{s}}$ by $v_{*}^{0}$. Thus, the droplet flux from an individual crest (30) reads

$$
\begin{aligned}
& F_{0 \mathrm{~s}}=\int_{r<r_{0}} \hat{F}_{0 \mathrm{~s}} \mathrm{~d} r, \\
& \hat{F}_{0 \mathrm{~s}}=3 c_{s} v_{*}^{0} r_{0}^{-3} r^{2},
\end{aligned}
$$

with the maximum radius of droplets $r_{0}$ defined by

$$
r_{0}=c_{r}\left(\gamma \nu / k_{\mathrm{b}}\right)^{1 / 3} u_{*}^{-1},
$$

where $c_{s}$ and $c_{r}$ are tuning constants (note that $\rho_{\mathrm{a}} / \rho_{\mathrm{w}}$ is absorbed in $c_{s}$ ).

The model of the spume-droplet production (28) with (32) reads

$$
V_{\mathrm{s}}(z)=k_{\mathrm{b}}\left(u_{*} / c_{\mathrm{b}}\right)^{3} \varsigma^{-5 / 2} \int_{r<r_{0}} \hat{F}_{0 \mathrm{~s}} \mathrm{~d} r,
$$

at $\varsigma>1$, and $V_{\mathrm{s}}=0$ at $\varsigma<1$, where $\varsigma=k_{\mathrm{b}} z$ is the dimensionless height, $c_{\mathrm{b}}=c\left(k_{\mathrm{b}}\right)$ is the phase velocity of the shortest breaking waves. To derive this relation, we took into account that the wavenumber of the shortest breaking waves is much larger than the spectral peak wavenumber, i.e. $k_{\mathrm{b}} \gg k_{\mathrm{p}}$. In (35), $r_{0}$ is defined by (34), and the spectrum of the droplet generation $\hat{F}_{0 \mathrm{~s}}$-by $(33 \mathrm{a}, 33 \mathrm{~b})$. Correspondingly, the total droplet volume flux $F_{\mathrm{s}}$ (or the model spray-generation function) is defined via $V_{\mathrm{s}}$ by Eq. 4 and reads

$$
F_{\mathrm{s}}(z)=(2 / 3)\left(u_{*} / c_{\mathrm{b}}\right)^{3} \varsigma_{1}^{-3 / 2} \int_{r<r_{0}} \hat{F}_{0 \mathrm{~s}} \mathrm{~d} r,
$$

where $\varsigma_{1}=\max (\varsigma, 1)$. The wind-speed dependence of $F_{\mathrm{S}}$ is proportional to $u_{*}^{4}$, and the flux rapidly attenuates with height on the scale of $1 / k_{\mathrm{b}}$.

\subsubsection{Range of Breaking Waves Producing Spume Droplets}

The generation of spume droplets is associated with the atomization of whitecaps and foam on breaking wave crests. The phase velocity of breaking waves generating whitecaps is larger then about $1.5 \mathrm{~m} \mathrm{~s}^{-1}$ (Gemmrich et al. 2008). Therefore, the upper limit of breaking waves that produce spume droplets $k_{\mathrm{b}}$ should be $k_{\mathrm{b}} \sim 5 \mathrm{rad} \mathrm{m}^{-1}$. On the other hand, the main contribution to $\Lambda(\mathbf{k})$ comes from shorter waves (see (32)) that break without air entrainment. The generation of droplets from these waves can arise if their breaking crests are disrupted by 

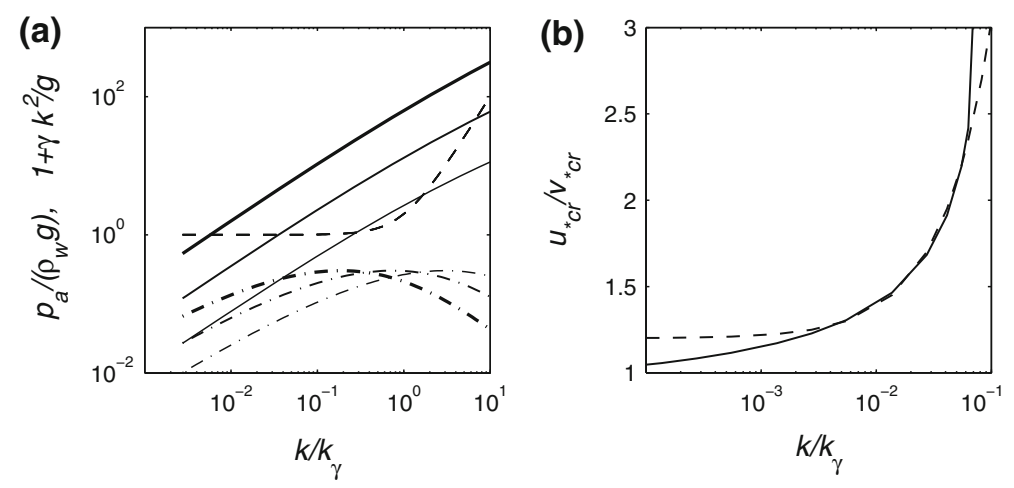

Fig. 2 a Scaled restoring force $1+\gamma k^{2} / g$ (r.h.s. of (37) shown by dashed line) and the depression of the aerodynamic pressure $p_{\mathrm{a}} /\left(\rho_{\mathrm{ag}}\right)$ over wave crests (1.h.s. of (37) shown by solid and dash-doted lines) versus $k / k_{\gamma}$ for different values of the friction velocity. Dashed-dotted lines show depression of the aerodynamic pressure over 'rough' surface wave crests with $z_{0}$ described by (9), and solid lines show the aerodynamic pressure depression over the smooth surface wave crests with $z_{0}$ described by (38). Thin, thick and very thick solid and dash-dotted lines show the aerodynamic pressure at a friction velocity $0.5,1$ and $2 \mathrm{~m} \mathrm{~s}^{-1}$ correspondingly. b Solid line: solution of equation (40) defining the critical friction velocity $u_{* \mathrm{cr}}$ in the MABL at which the crest of a breaking wave with the wavenumber $k$ should be disrupted by the Kelvin-Helmholtz instability and atomized into the spume droplets. Dash line: parametrization (41). $v_{* \mathrm{cr}}=0.45 \mathrm{~m} \mathrm{~s}^{-1}$ is the critical friction velocity over a smooth surface

the aerodynamic forcing. This problem can be treated within the framework of the KelvinHelmholtz instability, investigated by Miles (1957) for shear flow over the surface waves. The Kelvin-Helmholtz instability appears when the depression of the surface pressure over wave crests exceeds the restoring force due to the action of gravity and the surface tension. Assuming that the mean wind profile is logarithmic, the critical condition for the slow (relative to the wind speed) surface waves reads (Phillips 1977, Eq. 4.3.32):

$$
\frac{\rho_{\mathrm{a}}}{\rho_{\mathrm{w}}} \frac{k u_{*}^{2}}{\kappa g} \int_{0}^{\infty} \ln ^{2}\left(1+z / z_{0}\right) e^{-k z} \mathrm{~d}(k z)=1+\frac{\gamma k^{2}}{g}
$$

where $\gamma$ is the surface tension. The r.h.s. of (37) describes the restoring force and the 1.h.s. is the depression of the aerodynamic pressure over the wave crest.

Each side of this equation, with $z_{0}$ described by the Charnock relation (9), is shown in Fig. 2a. In this case, the restoring force (shown by the dashed line) exceeds the aerodynamic pressure for the rough surface at any wind speed and for all wave components (see dash-dotted lines), i.e. the Kelvin-Helmholtz instability is suppressed under all conditions. Nevertheless we note that the instability may emerge if the Charnock constant $c_{*}$ in (9) is decreased about 10 times (not shown here).

On the other hand, the Kelvin-Helmholtz instability emerges (the aerodynamic pressure exceeds the restoring force, the dash and solid lines in Fig. 2a are overlapped) if the surface is aerodynamically smooth with $z_{0}$ described by

$$
z_{0}=c_{v} v / u_{*}
$$

where $v$ is the air viscosity and $c_{v}=0.1$. The instability appears at $u_{*} \simeq 0.45 \mathrm{~m} \mathrm{~s}^{-1}$ in the vicinity of the wavenumber $k \simeq k_{\gamma}$, where $k_{\gamma}=(g / \gamma)^{1 / 2}$. At higher wind speeds the 
Kelvin-Helmholtz instability is spread over the wider wavenumber range (i.e. the interval of overlapping of dash and solid lines becomes wider for the higher wind speed).

The question is raised-is the Kelvin-Helmholtz instability relevant for the dropletgeneration problem if the calculations presented in Fig. 2a indicate stability of the sea surface with $z_{0}$ defined by (9)? The answer was given by Koga (1981) who provided convincing experimental evidence on the efficiency of the Kelvin-Helmholtz instability mechanism in the direct production of droplets at the crests of breaking waves.

Certainly, $z_{0}$ defined by (9) parametrizes the form drag supported by the full range of wind waves. On the other hand, the local surface aerodynamic roughness is very irregular, and different surface patterns have very different local roughness. This, in the first place, is relevant for the windward slopes and crests of breaking waves that can be considered as a locally 'smooth' patterns. We may anticipate that the airflow over such locally smooth areas accelerates and may create favorable conditions for the development of the Kelvin-Helmholtz instability of the small-scale surface disturbances, which inevitably cover windward slopes and the crests of breaking waves.

To simulate this process we use a two-layer approximation of the turbulent boundary layer over a surface wave suggested by Kudryavtsev et al. (2001) and Kudryavtsev and Makin (2004), which is based on the rapid distortion theory by Belcher and Hunt (1993). The airflow above a wave is divided into the outer region, $z>l$, where the wave-induced undulations are almost 'inviscid', and the inner region, $z<l$, where the wave-induced variation of the turbulent characteristics are confined. For the 'slow' surface waves, the inner region scale is $k l \sim 0.1$. The total wind velocity, $U_{l}$, (sum of the mean and the wave-induced undulation) over the wave crest at $z=l$ approximately reads (see e.g. Eq. A23 from Kudryavtsev and Makin (2004)):

$$
U_{l}=(1+\epsilon)\left(u_{*} / \kappa\right) \ln \left(l / z_{0}\right)+2 \epsilon\left(u_{*} / \kappa\right) \ln [1 /(k l)],
$$

where $\epsilon$ is the wave steepness and $u_{*}$ is the mean friction velocity in the outer region. The wind velocity profile inside the inner region over the locally smooth surface (with $z_{0 v}$ defined by (38)) has the logarithmic profile, $U(z)=\left(v_{*} / \kappa\right) \ln \left(z / z_{0 v}\right)$, which must be patched with (39). This gives the following resistance law for the friction velocity, $v_{*}$ in the inner region:

$$
\frac{u_{*}}{v_{*}}=\frac{\ln \left(l / z_{0 v}\right)}{(1+\epsilon) \ln \left(l / z_{0}\right)+2 \epsilon \ln [1 /(k l)]} .
$$

We shall use this equation in order to assess the critical friction velocity, $u_{* \mathrm{cr}}$, in the MABL at which crests of small-scale breaking waves (not carrying white caps) can be disrupted aerodynamically. The critical friction velocity $v_{* c r}$ in the shear flow over the smooth surface is about $v_{* \mathrm{cr}} \approx 0.45 \mathrm{~m} \mathrm{~s}^{-1}$ and unstable perturbations have $k$ around $k_{\gamma}$ (see Fig. 2a). Therefore, substituting in (40) $v_{* \mathrm{cr}}$ as $v_{*}$ and $z_{0 v}\left(v_{* \mathrm{cr}}\right)$ as $z_{0 v}$ we obtain the equation for the determination of $u_{* \mathrm{cr}}$ as a function of the breaking wave wavenumber $k$ and its steepness $\epsilon$. At this value of $u_{*}$ small-scale disturbances covering breaking waves with the wavenumbers $k$ are disrupted by the Kelvin-Helmholtz instability and then can be atomized into the droplets. The dependence of $u_{* \mathrm{cr}}$ on the wavenumber of breaking waves $k$ with $\epsilon=0.3$ is shown in Fig. $2 \mathrm{~b}$. In the range of long waves $\left(k / k_{\gamma}<10^{-3}\right), u_{* \mathrm{cr}} \simeq v_{* \mathrm{cr}}$, the critical value of the $10-\mathrm{m}$ wind speed is about $u_{10 \mathrm{cr}}=13 \mathrm{~m} \mathrm{~s}^{-1}$. Instability on the crests of shorter breaking waves appears at higher wind speeds, and crests of breaking waves with $k / k_{\gamma}>7 \times 10^{-2}$ are stable at any wind speeds. For a practical purpose, calculations shown in Fig. $2 b$ can be approximated as $u_{* \mathrm{cr}} / v_{* \mathrm{cr}}=1.2+18.2\left(k / k_{\gamma}\right)$, which is shown by the dashed line. This expression can be rewritten in terms of the upper limit $k_{\mathrm{wb}}$ of the range of wind waves whose breaking crest can be disrupted aerodynamically and then atomized into the droplets: 


$$
k_{\mathrm{wb}} / k_{\gamma}=\min \left[5.5 \times 10^{-2}\left(u_{*} / v_{* \mathrm{cr}}-1.2\right), 0.07\right] .
$$

We have assumed already that the production of spume droplets is associated with the atomization of white caps generated by breaking waves with $k<k_{\mathrm{b}} \simeq 5 \mathrm{rad} \mathrm{m}^{-1}$. Therefore, the aerodynamical disruption of crests of shorter breaking waves will extend the range of waves producing spume droplets. We define the upper limit of breaking waves producing spume droplets (primary parameter of the spray-generation model (36) with (33a, 33b) and (34)) as follows:

$$
k_{\mathrm{b}}=\max \left(k_{\mathrm{wc}}, k_{\mathrm{wb}}\right)
$$

where $k_{\mathrm{wc}}=5 \mathrm{rad} \mathrm{m}^{-1}$ is the wavenumber of the shortest breaking waves generating white caps, and $k_{\mathrm{wb}}$ is defined by (41).

\subsection{Comparison with Observations}

The magnitude of the spray-generation function predetermines the efficiency of the impact of sea spray on the MABL dynamics (see, e.g. the approximate solution (24) and (25)). Therefore, in order to obtain reliable results, the model spray-generation function should be consistent with observations. A comprehensive review of the available empirical spume spray-generation functions is given by Andreas (2002). It can be seen that the empirical $\hat{F}_{\text {s }}$ differ from each other by several orders (more than 5) of magnitude. A plausible cause for such a difference is that all functions are based on measurements taken in a limited range of the radius, the wind speed and at different heights above the sea level. All of them are extrapolated then to a larger radius, larger wind speed and to the surface using some heuristic arguments.

An example of the empirical spray-generation functions, proposed by Andreas (1998) and Smith and Harrison (1998), in terms of the spectrum of droplets concentration at the surface, $\hat{s}=\hat{F}_{\mathrm{s}} / a$, for the wind speed of $30 \mathrm{~m} \mathrm{~s}^{-1}\left(u_{*}=1.4 \mathrm{~ms}^{-1}\right)$, is shown in Fig. 3a. Both functions have a rapid cut-off at droplets radii $r>100 \mu \mathrm{m}$. This feature may be explained by the fact that the measurements of spray were made well above the surface whereto droplets of the large radius (and thus, of the large terminal fall velocity) cannot be transported by turbulence from the spray-generation layer. Therefore, we argue that the data shown in Fig. 3a do not provide a reliable information on the shape of $\hat{F}_{\mathrm{S}}$ at $r>100 \mu \mathrm{m}$.

Results of the extensive measurements of the spray generation in laboratory conditions were recently reported by Fairall et al. (2009). The measured spectra of the droplet concentration at a height of $z=0.125 \mathrm{~m}$ above the mean water surface at friction velocities $u_{*}=1.35$, 1.44 and $1.64 \mathrm{~m} \mathrm{~s}^{-1}$ are shown in Fig. 3b. First we note that, in the range $r<100 \mu \mathrm{m}$ the concentration of droplets measured under laboratory conditions has the same order of magnitude as predicted by the empirical spray-generation functions by Andreas (1998) and Smith and Harrison (1998). In the range of larger droplet size $(r>100 \mu \mathrm{m})$, the laboratory measurements differ significantly from the empirical function, and demonstrate almost constant level of the spray concentration up to the radius of the largest measured droplets. No indication on the existence of the spectral cut-off can be found in Fig. 3b.The laboratory measurements of Koga (1981) and Anguelova et al. (1999) revealed the generation of spume droplets with the radius of a few mm. Therefore, we may anticipate that the range of spume droplets at a wind speed of about $30 \mathrm{~m} \mathrm{~s}^{-1}$ can be extended up to $10^{-3}-2 \times 10^{-3} \mathrm{~m}$. It is noted also that the spume-droplet generation function recently developed by Mueller and Veron (2009) has a spectral cut-off that depends on the wind speed and varies from about $10^{-2} \mathrm{~m}$ at $U_{10}=10 \mathrm{~m} \mathrm{~s}^{-1}$ to about $10^{-3} \mathrm{~m}$ at $U_{10}=60 \mathrm{~m} \mathrm{~s}^{-1}$ (see their Fig. 2d). 

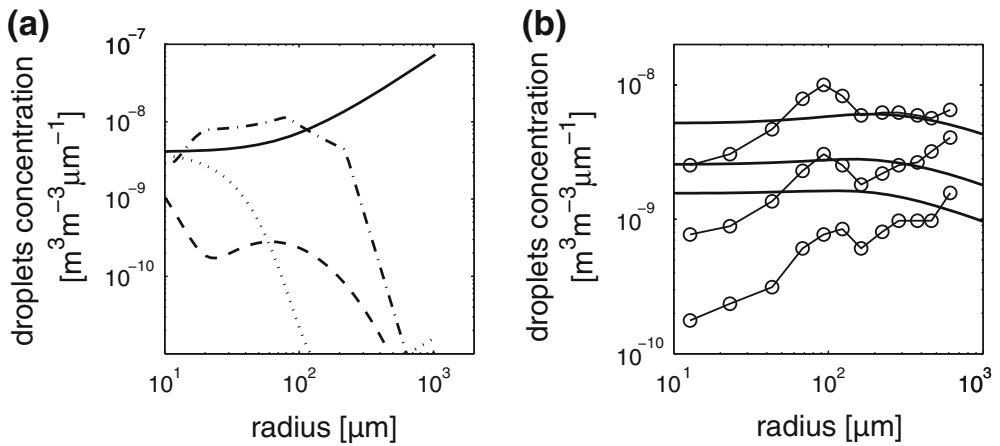

Fig. 3 a Spray-generation function scaled by the terminal fall velocity as a function of the droplet radii for $U_{10}=30 \mathrm{~m} \mathrm{~s}^{-1}$. Dashed line, Smith and Harrison (1998); dashed-dotted line, Andreas (1998); solid line, the model. Dotted line indicates the model concentration of droplets at $z=10 \mathrm{~m}$ (a 'simulation' of spray-generation function). $\mathbf{b}$ The concentration of droplets at $z=0.125 \mathrm{~m}$ as a function of their radius for $u_{*}=1.35,1.44$ and $1.64 \mathrm{~m} \mathrm{~s}^{-1}$ (shown by the curves of the same style from down to top). Solid lines with open circles are laboratory data by Fairall et al. (2009) taken from their Fig. 7, upper plot. Solid lines are model simulations of these measurements

The data shown in Fig. 3 are used to determine the tuning constants $c_{s}$ and $c_{r}$ in relations (33a, 33b) and (34) that define the model spray-generation function (36). In order to fit the model cut-off $r_{0}$ to the data reported by Koga (1981) and Anguelova et al. (1999) (as well as to be consistent with the spume-generation function of Mueller and Veron (2009)), the constant $c_{r}$ is fixed at $c_{r}=4.5$, which gives the spectral cut-off of spume droplets of about $r_{0}=1 \mathrm{~mm}$ at $U_{10}=30 \mathrm{~m} \mathrm{~s}^{-1}$. The other tuning constant $c_{s}$ was fixed at $c_{s}=1.4 \times 10^{-5}$ in order to fit the level of the empirical spray-generation functions shown in Fig. 3a in the spectral range $r<100 \mu \mathrm{m}$.

The model flux $\hat{F}_{\mathrm{S}}$ at the surface calculated according to (36) and scaled by the fall velocity $a$ is shown in Fig. 3a. The model spectrum of the droplet concentration at $z=10 \mathrm{~m}$, calculated according to (22) with (36), is also shown. The latter spectrum exhibits a cut-off of the concentration of droplets with $r>50 \mu \mathrm{m}$; these droplets due to a large terminal fall velocity cannot be transported upward by turbulence from the spray-generation layer. If this model spectrum $\hat{s}(r, 10 \mathrm{~m})$ would be erroneously treated in terms of the spray-generation function (assuming that $\hat{F}_{\mathrm{s}}=a \hat{s}$ ), then one might find its similarity with the empirical $\hat{F}_{\mathrm{s}}$.

In order to check the validity of the model against the laboratory data shown in Fig. 3b, the spectral density of wave breaking fronts described by (32) was confined to the wavenumber range from $k_{\mathrm{b}}$ to $k_{\mathrm{p}}$, where $k_{\mathrm{p}}$ is the wavenumber of the spectral peak given in Table 1 of Fairall et al. (2009). The model calculations of $\hat{s}$ at $z=0.125 \mathrm{~m}$ with fixed above values of the tuning constants $c_{r}$ and $c_{s}$ are shown in Fig. 3b. It is noted that the spectral cut-off of the model droplet spectra varies from 980 to $1100 \mu \mathrm{m}$. In the range $r>100 \mu \mathrm{m}$, the model overall is consistent with the data that may justify the validity of the spray-generation model. It is noted that the spray-generation function as reported by Fairall et al. (2009) is strongly wind speed dependent; the wind-speed exponent of the spray concentration in their experiments varies from 4.5 to 13 (see also the wind-speed dependence in Fig. 3b). Though the integral production of droplets in our model is proportional to $u_{*}^{4}$, its spectral production is proportional to $u_{*}^{7}$, which does not contradict the data (see e.g. (36) with (33a, 33b) and (34)).

The model spray-generation function (36) integrated over all droplet sizes is shown in Fig. 4. At the sea surface the generation function has two distinct regimes, at $u_{*}<0.7 \mathrm{~m} \mathrm{~s}^{-1}$ and $u_{*}>1 \mathrm{~m} \mathrm{~s}^{-1}$. In the former case, the spume droplets are produced by breaking waves 


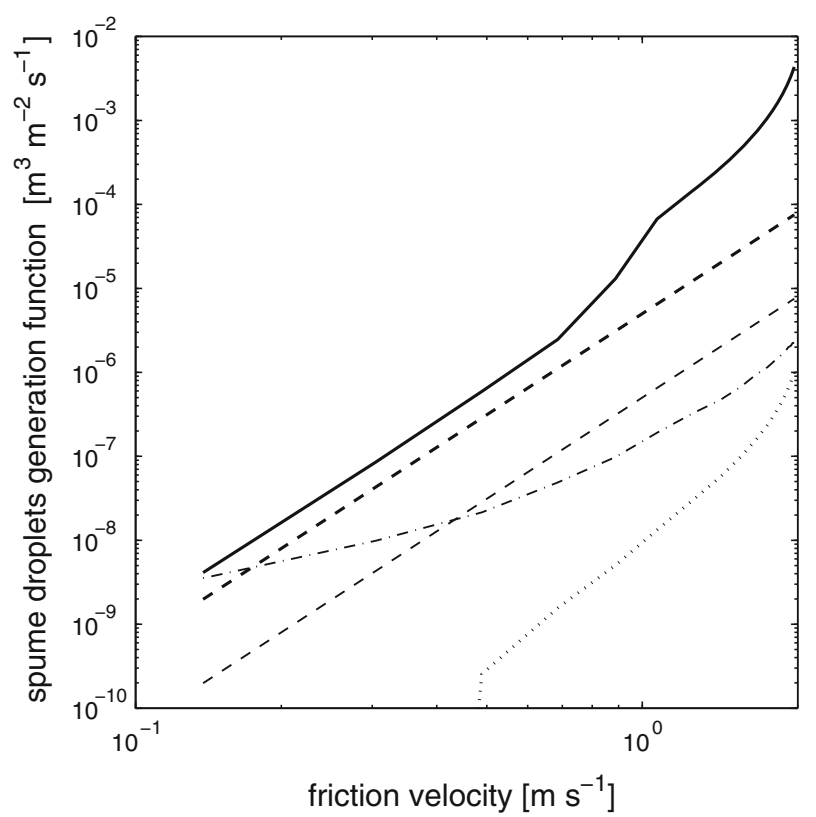

Fig. 4 Spume droplet-generation function as a function of the friction velocity. Thin and thick dash lines are spray-generation function by Andreas (1998) integrated over spume droplets $(r>20 \mu \mathrm{m})$ and its 'heuristic' version obtained by multiplication by a factor of 10. Solid line, dash-dotted and dotted lines are the integral model spray-generation function (36) at the surface, on the height of standard deviation of wind waves and on the standard 10-m level correspondingly

carrying the white caps, and in the latter case, by wider range of breaking waves due to the aerodynamic disruption of small-scale breaking wave crests by the Kelvin-Helmholtz instability, as predicted by (42). The aerodynamic disruption of small-scale breaking crests results in an explosive enhancement of the droplet production. At the sea surface the model exceeds empirical spray-generation function suggested by Andreas (1998) which was integrated over the spume droplet sizes $(r>20 \mu \mathrm{m})$. The reason for such a discrepancy has been already discussed above: the empirical function is built on the measurements made at different levels above the surface where the large spume droplets cannot be traced and measured. The model spray-generation function at the altitude of the standard deviation of the sea-surface displacement (for the given fetch of $300 \mathrm{~km}$ ) and at a standard 10-m level (also shown in Eq. 36 as an example) confirm this fact, and is consistent with the empirical function. It is noted also that the model agrees with a 'heuristic' spray-generation function used by Andreas and Emanuel (2001) for the estimate of the effect of spray on tropical cyclone intensity.

\subsection{Solution of the Coupled Sea Droplet-Atmosphere Model}

In this section, we consider the solution of the MABL model (18), (20) and (22) with the spray-generation function defined by (36). The system of equations is solved by iterations.

The wind-velocity profile for $U_{h}=70 \mathrm{~m} \mathrm{~s}^{-1}$, where $U_{h}$ is the wind speed at the reference level $h=100 \mathrm{~m}$, the vertical distribution of the dimensionless wind shear and the local kinematic turbulent stress defined by (18), (27) and (21) correspondingly, are shown in Fig. 5a-c)). Horizontal dotted lines in this figure indicate the aerodynamic roughness length $z_{0}$ and the 

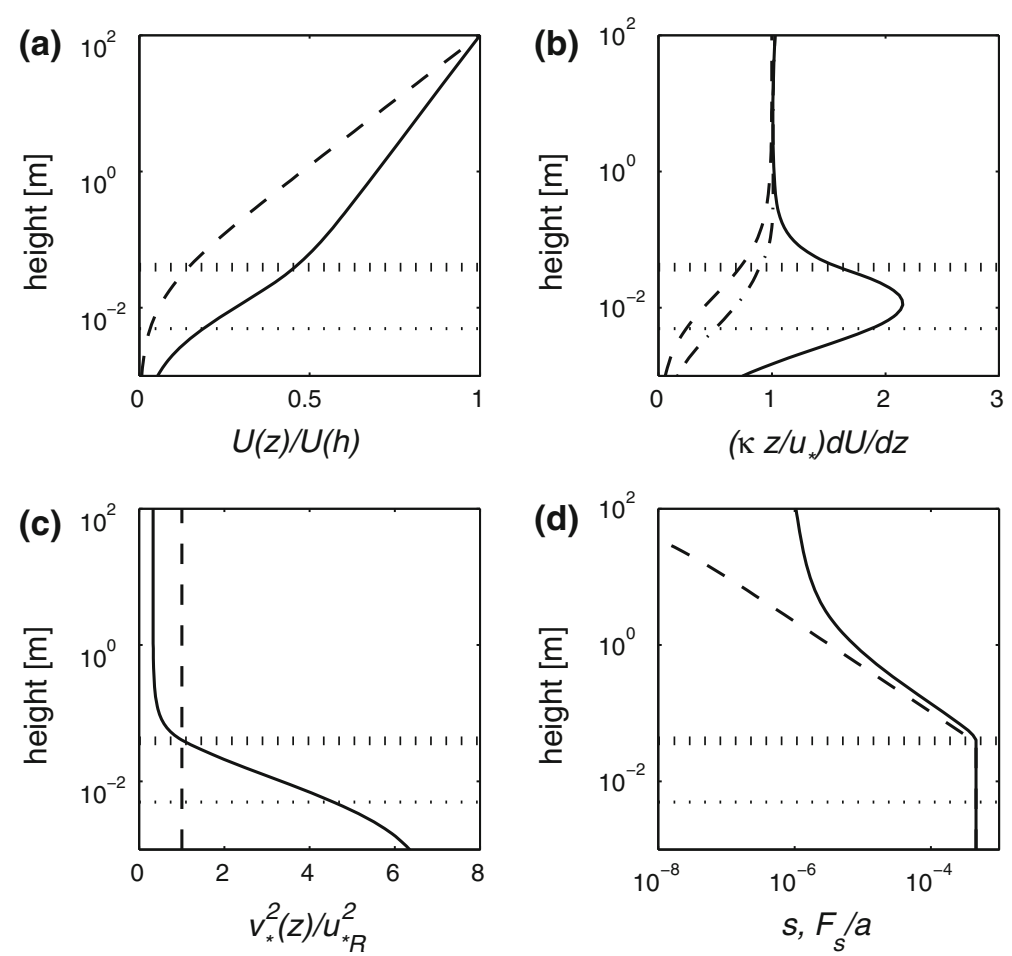

Fig. 5 a Wind profile $U(z) / U(h)$; solid line, with spray; dashed line, without spray. b Profile of the dimensionless wind shear defined by (27); solid line, with spray; dashed line, without spray; dashed-dotted line, the spray force is switched off. $\mathbf{c}$ The kinematic stress $v_{*}^{2} / u_{* R}^{2}$ profile defined by (21) and scaled by reference (without spray) friction velocity $u_{* R}$. d Profile of the droplet concentration $s$, solid line; profile of the dimensionless flux of droplets due to the wind tearing off breaking wave crests $s_{*}=\int\left(\hat{F}_{\mathrm{S}} / a\right) \mathrm{d} r$, dashed line. Horizontal dotted lines in plots (a)-(d) indicate height of the aerodynamic roughness $z_{0}$ (thin dotted lines) and altitudes $z=1 / k_{\mathrm{b}}$ (thick dotted lines). $U_{h}=70 \mathrm{~m} \mathrm{~s}^{-1}$, which is equivalent to $u_{10}=55 \mathrm{~m} \mathrm{~s}^{-1}$

altitude of breaking crests of shortest breaking waves $\left(z=1 / k_{\mathrm{b}}\right)$. Comparing dashed (no spray effect) and solid lines in these plots, one can notice that the effect of droplets leads to a significant deviation of the turbulent airflow structure from its reference (without spray impact) one.

First we mention that above the spray-generation layer the turbulent stress is decreased by a factor of four while at the lower bound of the MABL it is enhanced by a factor of six to seven relative to the reference value, Fig. $5 \mathrm{c}$. Increase of the near-surface stress is the consequence of the action of the force required to tear off the droplets from breaking crests and to accelerate them to the wind velocity. Then being injected at the altitude of breaking crests, the droplets fall and accelerate the airflow in the spray-generation layer due to the action of the spray vortex force caused by interaction of the 'spray rain' with the wind shear described by the r.h.s. of (3). This acceleration is well expressed in terms of the wind velocity shear shown in Fig. 5b. Owing to the continuity of the wind velocity, acceleration of the airflow in the spray-generation layer leads to an acceleration of the airflow above (see Fig. 5a). This, in turn, results in a decrease (relative to the reference value) of the wind shear above the spray-generation layer and thus, in the suppression of turbulence and the turbulent stress in this layer. It is noted that comparing the solid and dash-dotted lines in Fig. 5b one can find 
that the impact of the droplets on the wind shear via the effect of stratification (described by the first term in (27)) is much weaker than their impact via the 'spray-force' effect (described by the second term in (27)).

Figure 5d shows the vertical distribution of the droplet concentration $s$, the dimensionless flux of droplets due to the wind tearing off breaking wave crests $s_{*}=\int\left(\hat{F}_{\mathrm{S}} / a\right) \mathrm{d} r$. The maximum of the droplet concentration is located at the surface. The profile of $s_{*}(z)$ represents the approximate solution of the mass conservation equation resulting from the balance of the spume-droplet production by tearing off crests of breaking waves and their fall due to the gravitational force (see Eq. 23). The profile of $s(z)$ is close to $s_{*}(z)$ in the area, where most droplets are produced. Above this layer, the droplet concentration profile $s(z)$ deviates from $s_{*}(z)$ due to the increasing role of the turbulent transport of droplets. At a given wind speed, the concentration of droplets is large enough to affect the density of the air-water mixture; for this case the mixture density at the surface is about $\rho(0) / \rho_{\mathrm{a}} \approx 1.4$.

The spectrum of the droplet concentration at different heights above the surface is shown in Fig. 6. Very light droplets, characterized by the parameter $\omega \equiv a /\left(\kappa v_{*}\right) \ll 1$, penetrate the whole MABL, as predicted by the solution (22). Being generated by all breaking waves, light droplets are effectively transported upward by turbulence away from the layer, where they were generated. Therefore, the vertical distribution of light droplets does not depend on the vertical profile of $\hat{F}_{\mathrm{S}}(z)$, and the magnitude of the light droplet concentration is defined by a value of $\hat{F}_{\mathrm{S}}$ at the surface. The larger dimensionless terminal fall velocity $\omega$, the more rapid attenuation of the droplet concentration with height is. Large droplets with $\omega \gg 1$ are not effectively transported by turbulence. Their concentration essentially depends on the vertical profile of the spume-droplet production. The heaviest droplets at the surface dominate the concentration spectrum; their concentration rapidly decreases with height following the vertical profile of the spume-droplet flux. For considered conditions in the first $2 \mathrm{~m}$, the concentration of largest droplets decreases by two orders of magnitude, and above $20 \mathrm{~m}$, these droplets are not traceable.

A standard representation of the model results in terms of the drag coefficient at $10-\mathrm{m}$ height, $C_{\mathrm{d} 10}$, is shown in Fig. 7. The reference values, calculated using the Charnock relation (9), are shown by the dashed line. The drag coefficient starts to deviate from the reference run at the wind speed above $20 \mathrm{~m} \mathrm{~s}^{-1}$, and at $u_{10}>40 \mathrm{~m} \mathrm{~s}^{-1}$ it rapidly decreases to very low values. The friction velocity levels off at about $40 \mathrm{~m} \mathrm{~s}^{-1}$ and with a further increase in the wind speed tends to a constant value.

The model results are compared with data reported by Powell et al. (2003) and Powell (2006). We remind the reader that the experimental values of $C_{d 10}, u_{*}$ and $z_{0}$ were obtained from the wind profiles, measured by releasing GPS wind drop sondes. The profiles were analyzed in several layers to obtain reliable estimates; the estimates based on the 20-160 m surface layer are considered as most reliable. These estimates and the confidence limits are shown in Fig. 7. Powell et al. (2003) analyzed 331 profiles obtained by GPS sondes, dropped in 15 storms from 1997 to 1999 . Their analysis determined a leveling off of the surface stress at wind speeds exceeding $34 \mathrm{~m} \mathrm{~s}^{-1}$. Powell (2006) extends this analysis to 2664 GPS sondes profiles acquired in the period 1997-2005. The range of the wind speed was extended up to $80 \mathrm{~m} \mathrm{~s}^{-1}$. The extended dataset shows a decrease of the drag coefficient to a value of about $10^{-3}$ above a wind speed of $50 \mathrm{~m} \mathrm{~s}^{-1}$, which is in an agreement with the present model results. The model results are also in agreement with measurements of Jarosz et al. (2007) shown by the dotted line in the same figure. 


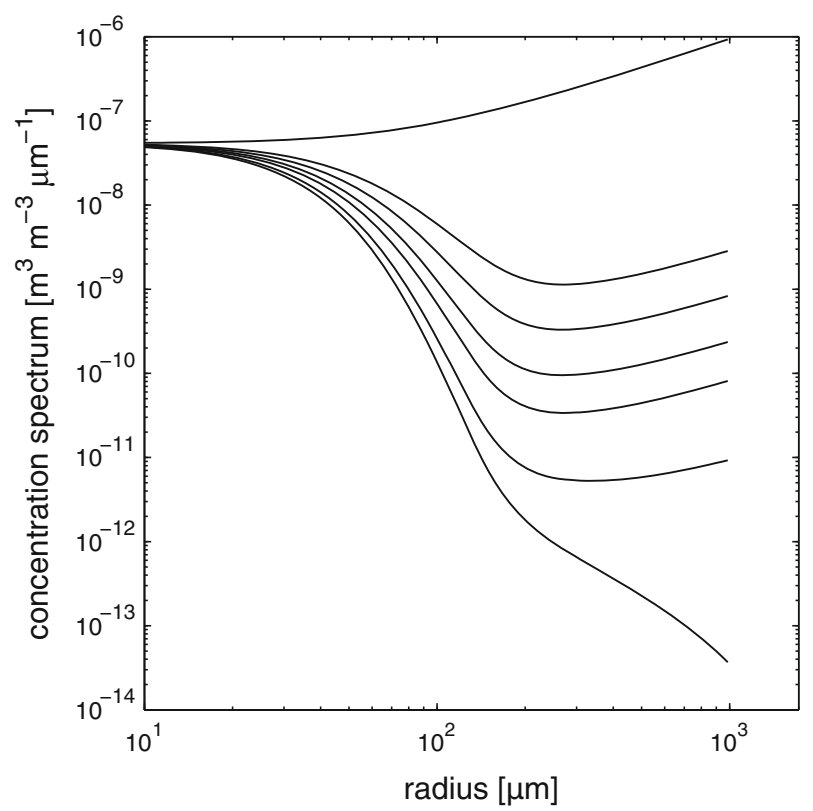

Fig. 6 Spectra of the droplet concentration at different heights; lines from top to bottom correspond to height: at the surface, $2,5,10,20,50$ and $100 \mathrm{~m} . U_{h}=70 \mathrm{~m} \mathrm{~s}^{-1}\left(U_{10}=55 \mathrm{~m} \mathrm{~s}^{-1}\right)$
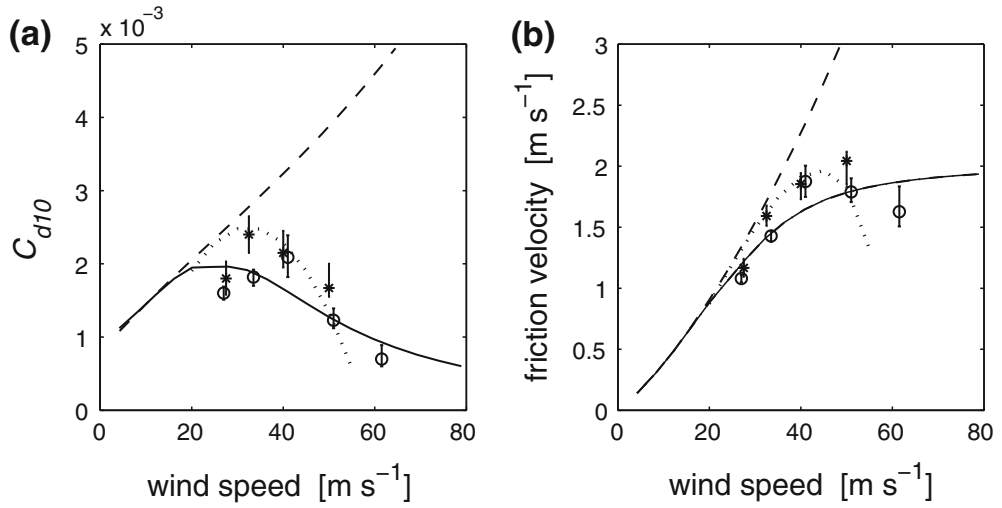

Fig. 7 a Drag coefficient and $\mathbf{b}$ friction velocity versus the wind speed at 10-m height. Solid line, according to the resistance law (20), droplets effects are accounted for; dashed line, reference run according to the Charnock relation (9), no droplet effects. Open circles, data by Powell (2006), compiled from his Fig. 7, layer 20-160 m; stars, data by Powell et al. (2003). compiled from their Fig. 3, layer 20-150 m; the $95 \%$ confidence limits on experimental estimates are indicated by vertical lines. Dotted line, fitted quadratic curve to the empirical data by Jarosz et al. (2007), their Fig. 3

\section{Discussion and Conclusions}

We have proposed a model describing the impact of ocean spray on the dynamics of the marine atmospheric surface boundary layer (MABL) in conditions of very high wind speeds. In this regime, sea droplets are torn off from breaking waves and are then injected into the airflow at the altitude of breaking wave crests. This phenomenological fact is taken into account in the 
mass and momentum conservation equations via the introduction of the 'volume sources'. The volume source of droplets (the rate of injection of droplets per unit volume of the air at height $z$ ) is used to investigate the spray-generation problem (e.g. Fairall et al. 1990, 2009; Kudryavtsev 2006). However, a similar term - the volume source of the droplet momentum (the rate of the injection of momentum of the droplets per unit volume of the air at height $z$ ) has never been (to our knowledge) taken into account in the moment conservation equation, and is included herein. Sea droplets affect the MABL dynamics in two ways: via the impact of droplets on the turbulent mixing through the stratification, and via the action of the spray force on the momentum of the spray-air mixture. The latter mechanism is described by the momentum conservation equation written in the non-Boussinesq approximation, where the effect of spray appears via the action of the 'vortex force' resulting from the interaction of the 'rain' of spray with the shear flow. The action of this 'vortex force' (called the 'sprayforce' effect) results in the acceleration of the airflow in the spray-generation layer and the reduction of the turbulent stress in the layer above. The 'stratification mechanism' enters the problem via the effect of droplets on the buoyancy force in the turbulent kinetic energy balance equation. It is parametrized using the Monin-Obukhov similarity theory for the stably stratified boundary layer. This mechanism also predicts suppression of the turbulent stress in the presence of spray.

The key element of the problem is the spray-generation function, which defines the vertical distribution of spray according to (22). As follows from the comprehensive review by Andreas (2002), the knowledge of the spray-generation function possesses great uncertainty. We found that sea droplets described by the empirical spray-generation function suggested by Andreas (1998) do not affect the MABL dynamics. On the other hand, if we assume that the empirical function underestimates the production of large droplets and extrapolate its spectral level to larger radii with the cut-off at $r=750 \mu \mathrm{m}$, then we find that the impact of sea droplets on the MABL dynamics becomes significant. In this case, droplets, through the effect of the 'spray force', result in the acceleration of the airflow and strong suppression of the surface drag at high wind speeds, while the efficiency of the 'stratification' mechanism is much weaker.

Other calculations were performed with the physical model of the spray-generation function proposed by Kudryavtsev and Makin (2009). The model assumes that droplets being torn off from crests of breaking waves are injected into the airflow at the altitude of breaking waves. The pulverization of the water/foam into droplets takes place in a thin boundary layer adjacent to each of the breaking wave crests. The production of droplets from an individual breaking crest is proportional to the wind speed, and the distribution of droplets over the size is proportional to $r^{2}$. The total volume production of droplets is proportional to the total length of the wave breaking fronts, where the main contribution comes from the shortest breaking waves (with wavenumber $k=k_{\mathrm{b}}$ ). At moderate wind speeds (less than $15 \mathrm{~ms}^{-1}$ ) $k_{\mathrm{b}} \simeq 5 \mathrm{rad} \mathrm{m}^{-1}$, which corresponds to the shortest breaking waves carrying white caps. At higher wind speeds, crests of shorter breaking waves can be disrupted by the wind (due to the Kelvin-Helmholtz instability) and the upper limit of breaking waves producing droplets extends to the range of small-scale breaking waves, up to $k_{\mathrm{b}} \simeq 25 \mathrm{rad} \mathrm{m}^{-1}$. The parameters of the model function were chosen so as to fit the empirical function of Andreas (1998) and laboratory data on the spray generation reported by Fairall et al. (2009), Koga (1981) and Anguelova et al. (1999).

Once parameters of the model spray-generation function are chosen, the solution of the boundary-layer model (Eqs. 18, 20 and 22) is obtained and compared with observations in terms of the drag coefficient. At this stage the main question arises: is the boundary-layer model capable of reproducing the fundamental experimental finding - the suppression of 
the surface drag at high wind speeds? The model calculations, presented in Fig. 7, confirm this capability. However, some details of the model, providing reasonable consistency of the model drag coefficient with data, should be clarified.

First, we mention that the solution of the coupled model shown in Fig. 5d confirms that the concentration of droplets near the surface approximately results from the balance of the droplet flux from breaking crests and their falling due to gravity. In addition, the efficiency of the effect of the stratification on the MABL dynamics is much weaker than the effect of the 'spray force' (see Fig. 5b). Thus, the approximate solutions (23), (24) and (25) are valid. Therefore, the drag coefficient (25) with the model function $F_{\mathrm{S}}$, defined by (36) with (33a, 33b), reads

$$
C_{\mathrm{d} h}=\kappa^{2}\left[\ln \left(\frac{h}{z_{0}}\right)+\frac{c_{s} \sigma}{6 \kappa^{2}} \frac{u_{*}^{3}}{c_{\mathrm{b}}^{3}} \frac{v_{*}^{0}}{u_{*}} \ln ^{2}\left(\frac{\delta}{z_{0}}\right)\right]^{-2} .
$$

where the effective depth of the spray-generation layer $\delta$ is defined as $\delta=3 / k_{\mathrm{b}}$. The second term in the denominator describes the impact of spray on the drag through the action of the 'spray force'. While the deviation of the friction velocity at the surface, $v_{*}^{0}$, from $u_{*}$ (we remind the reader that the ratio $v_{*}^{0} / u_{*}$ characterizes the effect of the airflow acceleration on the droplet production) remains small, i.e, $v_{*}^{0} / u_{*} \approx 1$, this term rapidly increases (as $u_{*}^{3}$ ) and leads to a decrease of $C_{\mathrm{d} h}$, as compared to the reference values (no spray effect). However, the suppression of $C_{\mathrm{d} h}$ is accompanied by the airflow acceleration that should stimulate additionally the droplet production described by the term $v_{*}^{0} / u_{*}$. Using (21) with (24) this ratio is approximately equal to

$$
v_{*}^{0} / u_{*}=\left(1-p_{s} u_{*}^{3} / c_{\mathrm{b}}^{3}\right)^{-1},
$$

where the parameter $p_{s}$ adsorbs other model parameters and constants:

$$
p_{s}=c_{s} \sigma /(3 \kappa) \ln \left(\delta / z_{0}\right) \text {. }
$$

Relation (44) shows that the effect of the acceleration can be important if the friction velocity approaches the saturated value

$$
u_{*}^{s}=p_{s}^{-1 / 3} c_{\mathrm{b}} .
$$

Relation (43) with (44) gives the following approximate solution for the drag coefficient:

$$
C_{\mathrm{d} h}=\kappa^{2}\left[\ln \left(h / z_{0}\right)+p_{s} /(2 \kappa) \ln \left(\delta / z_{0}\right)\left(u_{*} / c_{\mathrm{b}}\right)^{3}\left(1-p_{s} u_{*}^{3} / c_{\mathrm{b}}^{3}\right)^{-1}\right]^{-2} .
$$

This expression for $C_{\mathrm{d} h}$ reproduces the numerical solution, shown in Fig. 7, with an accuracy of $\pm 3 \%$. With increasing wind speed the second term in the denominator of (47) rapidly increases leading to a rapid decrease of the drag coefficient and to the leveling off of the friction velocity. It can be shown that at very high wind speeds the asymptotic solution of Eq. (47) is consistent with the saturation value of the friction velocity (46), and reads

$$
C_{\mathrm{d} h} \asymp p_{s}^{-2 / 3} c_{\mathrm{b}}^{2} / U_{h}^{2} .
$$

In this regime, the drag coefficient decreases as $U_{h}^{-2}$. This feature, as well as the saturation of the friction velocity, can be easily revealed in Fig. 7 at $U_{10}>40 \mathrm{~m} \mathrm{~s}^{-1}$. We may call this regime as the dynamics of a turbulent flow fully saturated with spume droplets. In this regime, the turbulent stress in the MABL has a limited saturated value that provides the balance between the production of spume droplets from breaking waves and the wind forcing providing the energy input to these breaking waves. In other words, in these conditions there 
is a negative feedback between the droplet production and the number of breaking crests wherefrom the droplets are torn, an increase/decrease of the droplet production leads to a decrease/increase of the friction velocity $u_{*}$ defining the intensity of wave breaking.

The approximate solution (47) clarifies the role and importance of the model tuning parameters that, we recall, are the constant $c_{s}$ defining the spectral level of the spray-generation function by (36) and (33a, 33b); $k_{b}$, the upper limit of breaking waves, providing the droplet generation and imposing the vertical distribution of the droplet flux; $c_{r}$, defining the maximal radius $r_{0}$ of droplets via (34). First, we mention that the solution does not depend on the cut-off radius $r_{0}$ of the droplet spectrum. Thus, the problem is only sensitive to the choice of $c_{s}$ determining the integral spume-droplet production. However, in our model treatment, the constant $c_{s}$ was interpreted as a constant defining the spectral level of the spray-generation function. In this respect, the discussion of the choice of $c_{r}$ is relevant. The value of $c_{r}$ was chosen so as to have maximum radius of droplets of order $10^{-3} \mathrm{~m}$ at a wind speed of about $U_{10}=30 \mathrm{~m} \mathrm{~s}^{-1}$, to be consistent with measurements of Koga (1981), Anguelova et al. (1999), and with the recently suggested spume-generation function proposed by Mueller and Veron (2009). The choice of $c_{s}$ and $k_{b}$ is much more important and, at the same time, uncertain. Once $c_{r}$ has been fixed, the parameters $c_{s}$ and $k_{\mathrm{b}}$ predetermine the spectral level and the vertical profile of the spray-generation function. The wavenumber $k_{\mathrm{b}}$ should be related to the shortest breaking waves that carry white caps and that can be 'easily' atomized into droplets by the wind forcing. These waves should be of order $k_{\mathrm{b}} \propto 5 \mathrm{rad} \mathrm{m}^{-1}$, as observed by Gemmrich et al. (2008). On the other hand we found that crests of shorter breaking waves (which are not accompanied by the generation of white caps) can be disrupted by the Kelvin-Helmholtz instability, and thus, can be also atomized into droplets. This mechanism extends the range of breaking waves generating spume droplets up to $k_{\mathrm{b}}=25 \mathrm{rad} \mathrm{m}^{-1}$. At fixed $k_{\mathrm{b}}$, the last tuning constant $c_{s}$ was chosen so as to fit the spectral level of the empirical spray-generation function suggested by Andreas (1998) in the range of droplet radii $10<r<100 \mu \mathrm{m}$, as well as to fit the data on the spray generation recently reported by Fairall et al. (2009). We have shown that the specified value of $c_{s}$ has the right order of magnitude.

Thus, the proposed model is capable of predicting the suppression of the surface drag at high wind speeds as a consequence of a reasonable choice of the model parameters related to the spray generation. The main impact of droplets on the boundary-layer dynamics arises from the effect of the 'spray force' on the momentum balance of the air-spray mixture. The efficiency of the impact of spray via the 'stratification effect' is significantly weaker. The 'spray force' effect originates from the action of the vortex force on the MABL dynamics; this force results from the interaction of the 'rain of spray' with the wind shear. This effect leads to the acceleration of the airflow and the suppression of the sea-surface drag. It is shown that the drag coefficient levels off at a wind speed of around $30 \mathrm{~m} \mathrm{~s}^{-1}$ and further decreases with increasing the wind speed as $U_{10}^{-2}$. This trend in $C_{d 10}$ is a consequence of the regime of the limited saturation of the MABL dynamics, when the friction velocity at very high winds levels off. These features are in agreement with recent experimental data of Powell et al. (2003), Powell (2006) and Jarosz et al. (2007) acquired in hurricanes. The model predictions on the saturation of the friction velocity in the MABL are consistent with field data on saturation of the radar backscattering in hurricanes observed by Donnelly et al. (1999).

Acknowledgments The ONR Grant N00014-08-1-0609, Federal Targeted Programme under the contracts, N02.740.11.5225 and N14.740.11.0201; and the EC FP7 project ERC PBL-PMES (contract 227915) are gratefully acknowledged. 
Open Access This article is distributed under the terms of the Creative Commons Attribution Noncommercial License which permits any noncommercial use, distribution, and reproduction in any medium, provided the original author(s) and source are credited.

\section{Appendix: Governing Mass and Momentum Conservation Equations}

The classical equations describing the dynamics of the turbulent flow with suspended heavy particles are introduced here following in general (Monin and Yaglom 1971, their Sect. 6.7). The only modification made is that we introduced 'volume sources' of the mass and the momentum simulating the injection of droplets into the airflow from the crests of breaking waves.

Volume source of spume droplets, $V_{\mathrm{s}}$, the total volume of droplets injected per unit time in unit volume of the air, is used to model the spume-droplet generation by the wind tearing off crests of breaking waves (Fairall et al. 1990, 2009; K06; KM09). Droplets, being torn off from breaking waves and accelerated to the wind velocity, are then injected into the airflow at the altitude of the crests of breaking waves. Therefore, as argued by Fairall et al. (1990, 2009) and K06, the generation of spume droplets should be included in the mass conservation equation as the volume source rather than the surface flux.

Introduction of the volume source of droplets leads to the straightforward modification of the original mass balance equation (Eq. 6.76 from Monin and Yaglom 1971) and the continuity equation (their Eq. 6.77), which take the form

$$
\begin{gathered}
\frac{\partial \rho}{\partial t}+\frac{\partial \rho v_{j}}{\partial x_{j}}=\rho_{\mathrm{w}} V_{\mathrm{s}}, \\
\frac{\partial}{\partial x_{j}}\left[u_{j}-s a \delta_{j 3}\right]=V_{\mathrm{s}} .
\end{gathered}
$$

Modification of the momentum conservation equation is also straightforward. To that end one needs, following the standard procedure, to sum up the equation describing the motions of the air and droplets, and then, taking into account the mass conservation equation (49), one arrives at the following horizontal momentum conservation equation:

$$
\frac{\partial}{\partial t}\left(\rho v_{\alpha}\right)+\frac{\partial}{\partial x_{j}}\left(\rho v_{\alpha} v_{j}+p \delta_{\alpha j}\right)=\rho_{\mathrm{w}} u_{\alpha} V_{\mathrm{s}}
$$

The term on the r.h.s. of this equation describes the volume source of the droplets momentum, the rate of injection of the droplet momentum in unit volume of the air at height $z$. If this term is omitted, then we arrive at the classical horizontal momentum conservation equation, see Eq. 6.79 in Monin and Yaglom (1971). The appearance of this term apparently results from violation of the 'continuity of the medium' introduced via the volume source of spray in (49). Notice also that Eq. 51 implicitly presumes that the droplets are injected into the airflow with a velocity equal to the air velocity at the height $z$.

In Eqs. 49-51, indexes $\alpha$ and $j$ possess the values $\alpha=1,2$ and $j=1,2,3 ; v_{j}$ is the mixture velocity defined via the air velocity $u_{j}$ and the terminal fall velocity of droplets $a$ as

$$
v_{j}=u_{j}-\frac{\rho_{\mathrm{w}} s}{\rho} a \delta_{j 3}
$$

and $p$ in (51) is the total pressure. 
Assuming stationary and spatially homogenous conditions, and that far above the ocean surface the droplet concentration and their flux vanish, the averaged equations (49) and (50) read

$$
\begin{aligned}
\overline{\rho v_{3}} \equiv \bar{\rho} \cdot \overline{u_{3}}-\rho_{\mathrm{w}} \bar{s} a+\triangle \rho \overline{s^{\prime} u_{3}^{\prime}} & =-\rho_{\mathrm{w}} F_{\mathrm{s}}, \\
\overline{u_{3}}-\bar{s} a & =-F_{\mathrm{s}},
\end{aligned}
$$

where the overbar denotes a mean value, $\overline{s^{\prime} u_{3}^{\prime}}$ is the turbulent flux of the droplet concentration, $\triangle \rho=\rho_{\mathrm{w}}-\rho_{\mathrm{a}}$, and $F_{\mathrm{S}}$ is the volume flux of droplets defined as

$$
F_{\mathrm{s}}\left(x_{3}\right)=\int_{x_{3}}^{\infty} V_{\mathrm{s}} \mathrm{d} x_{3} .
$$

Adding Eqs. 53 and 54 results in the mass conservation equation for droplets

$$
\overline{s^{\prime} u_{3}^{\prime}}-\bar{s} a=F_{\mathrm{s}}
$$

where condition $\bar{s} \ll 1$ is taken into account.

The averaged horizontal momentum conservation equation takes the form

$$
\frac{\partial}{\partial x_{3}}\left[\bar{\rho} \overline{u_{\alpha}^{\prime} u_{3}^{\prime}}+\bar{u}_{\alpha}\left(\bar{\rho} \cdot \bar{u}_{3}+\Delta \rho \overline{s^{\prime} u_{3}^{\prime}}-\rho_{\mathrm{w}} a \bar{s}\right)\right]=\rho_{\mathrm{w}} \bar{u}_{\alpha} V_{\mathrm{s}}
$$

where a term containing the third moment $\overline{\rho^{\prime} u_{\alpha}^{\prime} u_{3}^{\prime}}$, and terms containing horizontal fluxes of droplets $\bar{u}_{3} \Delta \rho \overline{s^{\prime} u_{\alpha}^{\prime}}$ and $\rho_{\mathrm{w}} a \overline{s^{\prime} u_{\alpha}^{\prime}}$, are neglected. Accounting for the mass conservation (53), equation (57) is reduced to

$$
\frac{\partial}{\partial x_{3}}\left(\bar{\rho} \overline{u_{\alpha}^{\prime} u_{3}^{\prime}}-\rho_{\mathrm{w}} \bar{u}_{\alpha} F_{\mathrm{s}}\right)=\rho_{\mathrm{w}} \bar{u}_{\alpha} V_{\mathrm{s}},
$$

and after some trivial reorganization we finally arrive at

$$
\frac{\partial}{\partial x_{3}}\left(\bar{\rho} \overline{u_{\alpha}^{\prime} u_{3}^{\prime}}\right)=\rho_{\mathrm{w}} F_{\mathrm{s}} \frac{\partial \bar{u}_{\alpha}}{\partial x_{3}} .
$$

The term on the r.h.s. of this equation can be interpreted as a 'vortex force' acting on the airflow due to vertical flux of the droplets through the velocity shear.

\section{References}

Andreas EL (1998) A new spray generation function for wind speeds up to $32 \mathrm{~m} / \mathrm{s}$. J Phys Oceanogr 28:21752184

Andreas EL (2002) A review of the sea spray generation function for the open ocean. In: Perrie W (ed) Atmosphere-ocean interactions, vol 1. WIT Press, Ashurst, pp 1-46

Andreas EL (2004) Spray stress revised. J Phys Oceanogr 34:1429-1440

Andreas EL, Emanuel KA (2001) Effect of sea spray on tropical cyclonic intensity. J Atmos Sci 58:3741-3751

Anguelova M, Barber RP, Wu J (1999) Spume drops produced by the wind tearing of wave crests. J Phys Oceanogr 29:1156-1165

Barenblatt GI (1953) On the motion of suspended particles in a turbulent flow. Prikl Mat Mekh 17:261-274

Barenblatt GI (1955) On the motion of suspended particles in a turbulent flow in a half-space or a plane open channel of finite depth. Prikl Mat Mekh 19:61-88

Barenblatt GI (1996) Scaling, self-similarity, and intermediate asymptotics. Cambridge University Press, Cambridge, UK, 386 pp

Barenblatt GI, Golitsyn GS (1974) Local structure of mature dust storms. J Atmos Sci 31:1917-1933 
Barenblatt GI, Chorin AJ, Prostokishin VM (2005) A note concerning the Lighthill sandwich model of tropical cyclones. Proc Natl Acad Sci USA 102:11148-11150

Belcher SE, Hunt JCR (1993) Turbulent shear flow over slowly moving waves. J Fluid Mech 251:109-148

Bintanja R (2000) Snowdrift suspension and atmospheric turbulence. Part I: Theoretical background and model description. Boundary-Layer Meteorol 95:343-368

Black PG, D'Asaro EA, Drennan WM, French JR, Niiler PP, Sanford TB, Terrill EJ, Walsh EJ, Zhang JA (2007) Air-sea exchange in hurricanes synthesis of observations from the coupled boundarylayer air-sea transfer experiment. Bull Am Meteorol Soc 88:357-374

Bridge JS, Dominic DF (1984) Bed load velocities and sediment transport rates. Water Resour Res 20:476-490

Bye JAT, Jenkins AD (2006) Drag coefficient reduction at very high wind speeds. J Geophys Res 111:C033024. doi:10.1029/2005JC003114

Donelan MA, Haus BK, Reul N, Plant WJ, Stiassnie M, Graber HC, Brown OB, Saltzman ES (2004) On the limiting aerodynamic roughness of the ocean in very strong winds. Geophys Res Lett 31:L18306. doi:10. 1029/2004GL019460

Donnelly WJ, Carswell JR, McIntosh RE, Chang PS, Wilkerson J, Marks F, Black PG (1999) Revised ocean backscatter models at $\mathrm{C}$ and Ku band under high-wind conditions. J Geophys Res 104:11485-11497

Emanuel KA (1995) Sensitivity of tropical cyclones to surface exchange coefficients and a revised steady-state model incorporating eye dynamics. J Atmos Sci 52:3969-3976

Fairall CW, Edson JB, Miller MA (1990) Heat fluxes, white caps, and sea spray. In: Geernaert GL, Plant WJ (eds) Surface waves and fluxes, vol 1. Kluwer, Dordrecht, pp 173-208

Fairall CW, Banner ML, Peirson WL, Asher W, Morison RP (2009) Investigation of the physical scaling of sea spray spume droplet production. J Geophys Res 114:C100001. doi:10.1029/2008JC04918

Gemmrich J, Banner M, Garrett C (2008) Spectrally resolved energy dissipation rate and momentum flux of breaking waves. J Phys Oceanogr 38. doi:10.1175/2007JPO3762.1

Jarosz E, Mitchell DA, Wang DW, Teague WJ (2007) Bottom-up determination of air-sea momentum exchange under a major tropical cyclone. Science 315:1707-1709

Koga M (1981) Direct production of droplets from breaking wind-waves-its observation by a multi-colored overlapping exposure photographing technique. Tellus 33:552-563

Kolmogorov AN (1949) Fragmentation of droplets in a turbulent flow. Doklady Akademii Nauk SSSR 66:825828

Kolmogorov AN (1954) On a new variant of the gravitational theory of motion of suspended sediments. Izv Akad Nauk SSSR Phys Ser 6:56-58

Komen GJ, Cavaleri L, Donelan M, Hasselmann K, Hasselmann S, Janssen PAEM (1994) Dynamics and modelling of ocean waves. Cambridge University Press, New York, $531 \mathrm{pp}$

Kudryavtsev VN (2006) On effect of sea droplets on atmospheric boundary layer. J Geophys Res 111:C07020. doi:10.1029/2005JC002970

Kudryavtsev VN, Makin VK (2004) Impact of swell on marine atmospheric boundary layer. J Phys Oceanogr 34:934-949

Kudryavtsev VN, Makin VK (2007) Aerodynamic roughness of the sea surface at high winds. Boundary-Layer Meteorol 125:289-303

Kudryavtsev VN, Makin VK (2009) Model of the spume sea spray generation. Geophys Res Lett 36:L06801. doi:10.1029/2008GL036871

Kudryavtsev VN, Makin VK, Meirink JF (2001) Simplified model of the airflow above waves. Boundary-Layer Meteorol 100:63-90

Kukulka T, Hara T, Belcher SE (2007) A model of the air-sea momentum flux and breaking-wave distribution for strongly forced wind waves. J Phys Oceanogr 37:1811-1828

Makin VK (2005) A note on drag of the sea surface at hurricane winds. Boundary-Layer Meteorol 115:169176

Melville WK, Matusov P (2002) Distribution of breaking waves at the ocean surface. Nature 417:58-62

Miles JW (1957) On the generation of surface waves by shear flow. J Fluid Mech 3:185-204

Monin AS, Yaglom AM (1971) Statistical fluid mechanics. MIT Press, Cambridge, 769 pp

Mueller JA, Veron F (2009) A sea state-dependent spume generating function. J Phys Oceanogr 39:2363-2372

Phillips OM (1977) Dynamics of the upper ocean. Cambridge University Press, New York, 336 pp

Powell MD (2006) Drag coefficient distribution and wind speed dependence in tropical cyclones. Final report to the National Oceanic and Atmospheric Administration, Joint Hurricane Testbed Program, Miami, $26 \mathrm{pp}$

Powell MD, Vickery PJ, Reinhold TA (2003) Reduced drag coefficient for high wind speeds in tropical cyclones. Nature 422:279-283

Smith MH, Harrison NM (1998) The sea spray generation function. J Aerosol Sci 29(Suppl 1):S189-S190 